\title{
Optimal Tradeoff between Sum-Rate Efficiency and Jain's Fairness Index in Resource Allocation
}

\author{
Akram Bin Sediq, Ramy H. Gohary, Rainer Schoenen, and Halim Yanikomeroglu
}

\begin{abstract}
The focus of this paper is on studying the tradeoff between the sum efficiency and Jain's fairness index in general resource allocation problems. Such problems are frequently encountered in wireless communication systems with $M$ users. Among the commonly-used methods to approach these problems is the one based on the $\alpha$-fair policy. Analyzing this policy, it is shown that, except for the case of $M=2$ users, this policy does not necessarily achieve the optimal Efficiency-Jain tradeoff (EJT). In particular, it is shown that, when the number of users $M>2$, the gap between the efficiency achieved by the $\alpha$-fair policy and that achieved by the optimal EJT policy can be unbounded, for the same Jain's index. Finding the optimal EJT corresponds to solving potentially difficult non-convex optimization problems. To alleviate this difficulty, we derive sufficient conditions, which are shown to be sharp and naturally satisfied in various radio resource allocation problems. These conditions provide us with a means for identifying cases in which finding the optimal EJT and the rate vectors that achieve it can be reformulated as convex optimization problems. The new formulations are used to devise computationally-efficient resource schedulers that enable the optimal EJT to be achieved for both quasi-static and ergodic time-varying communication scenarios. Analytical findings are confirmed by numerical examples.
\end{abstract}

\section{INTRODUCTION}

The resources available for wireless communication systems are usually scarce and shared among multiple users. The way in which these resources are allocated determines the efficiency of the system and the benefits received by its users. Since the service provider is interested in maximizing the system efficiency and the users are interested in maximizing their own benefits, the allocation of resources is typically encountered by conflicting goals. For instance, favouring a certain class of users may increase the system efficiency, but would result in the dissatisfaction of other classes of users. In contrast, providing equal benefits to all users may result in higher fairness, but will potentially result in low efficiency. To control the emphasis placed on various goals, the provider uses a tradeoff policy, which, unless properly chosen, can result in wasteful allocation of resources. In particular, a suboptimal tradeoff policy can be less efficient and, at the same time, less fair to the users [1]-[3].

The benefits received by the users in the downlink of a wireless communication system can be measured by the rates at which data is delivered to these users. These rates

The authors are with the Department of Systems and Computer Engineering, Carleton University, Ottawa, ON, Canada.

This work is supported, in part, by Huawei Technologies Canada Co., Ltd., in part, by the Natural Sciences and Engineering Research Council (NSERC) of Canada and, in part, by the Ontario Graduate Scholarship (OGS)

A preliminary version of this work was presented at 2012 IEEE Int. Symp. Personal, Indoor and Mobile Radio Commun. (PIMRC'12). are controlled by appropriate allocation of radio resources at the transmitter. For instance, the transmitter may allocate its resources in such a way that maximizes the sum of the rates delivered to the users. This allocation favours users that are geographically closer to the transmitter, but "starves" farther users, and although more efficient from the system perspective, such an allocation is unfair to the users at less advantageous locations [4], [5]. A fairer allocation is one in which the minimum rate received by the users is maximized [1], [3], [6]. However, this allocation can result in unacceptable system efficiency, i.e., low sum-rate. Hence, it is desirable to find an optimal tradeoff policy whereby the system provider allocates its resources in such a way that no other allocation provides a strictly higher efficiency and at the same time be fairer to the users. The focus of this paper is to develop a technique for obtaining an efficiency-fairness tradeoff that is optimal in a specific sense and to derive sufficient conditions, which, when satisfied by the set of feasible benefits, lead to efficiently computable optimal tradeoff and benefit vectors.

The applications that we will consider herein are derived from practical radio resource scheduling problems that arise in wireless communication systems operating over quasi-static and ergodic time-varying channels. However, our analysis applies to a broader class of frameworks, including social and economics ones [2], [7], [8].

To study the tradeoff between efficiency and fairness we note that efficiency is usually defined depending on the particular resource allocation problem considered. For instance, in the case of wireless networks considered herein, efficiency is measured by the sum-rate delivered to the users of the network. In contrast, several definitions are used to quantify fairness. In [2], axioms that include continuity and homogeneity, and subsequent features, are provided to obtain a general class of plausible fairness measures. Among the members of that class are the entropy-based index [2] and Jain's fairness index [9]. In addition to the axioms and the features provided in [2], we identify two more features that commend the use of Jain's index as a fairness measure.

- Conformity to standard fairness benchmarks: A fairness measure with this feature can be related to easily conceivable benchmarks. For instance, a Jain's index of $p / 100$ can be regarded as the fairness index of an equivalent resource allocation in which $p \%$ of the users receive equal non-zero benefits and the remaining $(100-p) \%$ receive zero benefits [9]. The relations between other metrics and standard benchmarks are not readily available.

- Accommodating more users: A good fairness measure enables more users with specific benefit requirements to 
be accommodated in the system. The superiority of Jain's index in that respect will be illustrated by numerical comparisons hereinafter.

A common approach to trading off efficiency with fairness in wireless networks, is to allocate the resources in a way that maximizes the sum-rate efficiency while ensuring that the minimum rates achieved by the users exceed some prescribed bounds, e.g., [3], [8]. Varying these bounds over the set of feasible rates provides a means for controlling fairness [3]. Another approach is to allocate the resources in a way that maximizes a parametric utility, whereby one, or multiple, parameters are used to control the emphasis on efficiency and fairness. A commonly used policy is the $\alpha$ fair one (also known as the $\alpha$-fair utility) [1], wherein various settings of a parameter $\alpha$ yield allocations that achieve popular efficiency-fairness tradeoffs. For instance, setting $\alpha=0$ yields maximum efficiency, setting $\alpha=1$ yields proportionally fair allocations [10], and setting $\alpha=\infty$ yields allocations that are fair in the max-min sense [1]. Motivations for using the $\alpha$-fair policy are provided in [2]. Generally speaking, increasing $\alpha$ results in allocations that are fairer [2] in a sense that does not necessarily conform to Jain's index, as will be shown hereinafter. Other parametric utilities for trading off efficiency and fairness are considered in [11] and [12], and a comparison between multiple tradeoff criteria is provided in [13].

Compared with other measures, Jain's index provides a fairness criterion that takes into consideration all the users of the system, not only those users that are assigned minimal resources [9]. Maximizing Jain's index without wasting valuable resources requires optimal tradeoff between efficiency and this index. A question that arises is whether maximizing the well-studied $\alpha$-fair policy yields such an optimal tradeoff. To address this question, we begin in this paper by showing that $\alpha$-fair allocations are not guaranteed to achieve the optimal Efficiency-Jain tradeoff (EJT) except for the case of $M=2$ users. To overcome this drawback, we develop a generic technique for obtaining optimal EJT allocations. Unfortunately, this technique involves solving potentially difficult nonconvex optimization problems. To alleviate this difficulty, we derive sufficient conditions, which are shown to be sharp and naturally satisfied in various radio resource allocation problems. These conditions provide us with a means for identifying cases in which finding the optimal EJT and the rate vectors that achieve it can be reformulated as convex optimization problems. The new formulations are used to devise computationally-efficient resource schedulers that enable the optimal EJT to be achieved for both quasi-static and ergodic time-varying communication scenarios.

Numerical results are provided for confirming our theoretical findings and for demonstrating the advantage of the optimal tradeoff provided by our technique over the $\alpha$-fair one.

Notation: Bold-face and regular-face fonts will be used to denote vectors and scalars, respectively. The set of length$M$ vectors with non-negative real entries will be denoted by $\mathbb{R}_{+}^{M}$ and the length- $M$ all-one and all-zero vectors will be denoted by $\mathbf{1}_{M}$ and $\mathbf{0}_{M}$, respectively. The symbols $\preceq$ and $\succeq$ will be used to denote element-wise inequalities, and $(\cdot)^{T}$ will be used to denote the transpose. The Euclidean norm will be denoted by $\|\cdot\|$.

\section{PRELIMINARIES}

Let $\mathbf{x} \in \mathcal{C} \subseteq \mathbb{R}_{+}^{M}$ be a vector of non-negative real entries $\left\{x_{m}\right\}_{m=1}^{M}$, where $x_{m}$ is the benefit received by user $m$ and $\mathcal{C}$ is the set of feasible benefit vectors. Generally, the benefits $\left\{x_{m}\right\}_{m=1}^{M}$ and the set $\mathcal{C}$ depend on the application and the resources allocated to each user [9, Sec. 5]. For example, in the downlink of wireless communications, $x_{m}$ can be the rate of user $m$ resulting from a particular allocation of the radio resources, and $\mathcal{C}$ is the set of all achievable rates. In this paper, the efficiency, $\eta(\mathbf{x})$, of a resource allocation is defined by the sum of benefits (i.e., $\eta(\mathbf{x})=\sum_{m=1}^{M} x_{m}$ ), and its fairness is given by the Jain's index defined below.

Definition 1 (Jain's Index). For $\mathrm{x} \in \mathbb{R}_{+}^{M}$, Jain's fairness index $J: \mathbb{R}_{+}^{M} \rightarrow \mathbb{R}_{+}$is given by [9]

$$
J(\mathbf{x})=\left(\sum_{m=1}^{M} x_{m}\right)^{2} / M \sum_{m=1}^{M} x_{m}^{2} .
$$

This definition shows that $J(\mathbf{x})$ is continuous and lies in $\left[\frac{1}{M}, 1\right]$. In this interval, $J=\frac{1}{M}$ corresponds to the least fair allocation in which only one user receives a non-zero benefit, and $J=1$ corresponds to the fairest allocation in which all users receive the same benefit.

In many cases, depending on $\mathcal{C}$, there is an inherent tradeoff between $\eta(\mathbf{x})$ and $J(\mathbf{x})$. Hence, to ensure efficient utilization of resources, we seek the optimal tradeoff, which is defined next.

Definition 2 (Optimal Efficiency-Jain tradeoff (EJT)). An optimal EJT is one that results in a benefit vector $\mathrm{x}^{\star}$ such that no $\mathbf{x} \neq \mathbf{x}^{\star}, \mathbf{x} \in \mathcal{C}$ that satisfies either: 1) $\eta(\mathbf{x})>\eta\left(\mathbf{x}^{\star}\right)$ and at the same time $J(\mathbf{x}) \geq J\left(\mathbf{x}^{\star}\right)$, or 2) $\eta(\mathbf{x}) \geq \eta\left(\mathbf{x}^{\star}\right)$ and at the same time $J(\mathbf{x})>J\left(\mathbf{x}^{\star}\right)$.

This definition is closely related to Pareto optimality defined for optimization problems with multiple objectives [14]. With efficiency and Jain's index as objectives, a Pareto optimal point is one at which efficiency cannot be increased without decreasing Jain's index and likewise, Jain's index cannot be increased without decreasing efficiency. As such, a point that is optimal from the EJT perspective, as per Definition 2, is equivalent to Pareto optimality in efficiency and Jain's index. However, a point that is Pareto optimal from an Efficiency-Jain perspective is not necessarily Pareto optimal if the multiple objectives are taken to be the users' benefits themselves, rather than the efficiency and Jain's index that these benefits achieve.

Definition 2 will be used in the next section to determine whether the $\alpha$-fair tradeoff policy achieves the optimal EJT.

\section{DOES $\alpha$-FAIR POLICY ACHIEVE THE OPTIMAL EFFICIENCY-JAIN TRADEOFF?}

Given an $\alpha \in[0, \infty)$, the benefit vector $\mathbf{x}_{\alpha}^{\star}$ generated by the $\alpha$-fair tradeoff policy maximizes the $\alpha$-fair utility [1], i.e.,

$$
\mathbf{x}_{\alpha}^{\star}=\arg \max _{\mathbf{x} \in \mathcal{C}} U_{\alpha}(\mathbf{x}),
$$


where

$$
U_{\alpha}(\mathbf{x})= \begin{cases}\sum_{m=1}^{M} \log x_{m}, & \alpha=1, \\ \frac{1}{1-\alpha} \sum_{m=1}^{M} x_{m}^{1-\alpha}, & \alpha \geq 0, \alpha \neq 1 .\end{cases}
$$

The $\alpha$-fair policy thus described was considered in [2]. It was shown therein that, for $\alpha \neq 1, \mathbf{x}_{\alpha}^{\star}$ generated by (2) is the same as that generated by

$$
\mathbf{x}_{\alpha}^{\star}=\arg \max _{\mathbf{x} \in \mathcal{C}}\left(\left|\frac{\alpha}{1-\alpha}\right| L\left(H_{\alpha}(\mathbf{x})\right)+L(\eta(\mathbf{x}))\right),
$$

where

$$
H_{\alpha}(\mathbf{x})=\operatorname{sgn}(1-\alpha) \sqrt[\alpha]{\sum_{m=1}^{M}\left(\frac{x_{m}}{\eta(\mathbf{x})}\right)^{1-\alpha}}
$$

and $L(\cdot) \triangleq \operatorname{sgn}(\cdot) \log (|\cdot|)$. This equivalent formulation of the $\alpha$-fair policy provides insight into the role of $\alpha$. In particular, it can be seen that $L(\cdot)$ is monotonically increasing and that, for any $\alpha \neq 1, H_{\alpha}(\mathbf{x})$ provides a homogeneous fairness measure [2]. Hence, it can be seen that increasing $\alpha$ places more emphasis on fairness at the expense of efficiency.

Using the above observations, it was argued in [2] that solving (4) yields a benefit vector that achieves the optimal tradeoff between $H_{\alpha}(\mathbf{x})$ and $\eta(\mathbf{x})$. Although this explanation offers a better understanding, it presents the fairness component of the $\alpha$-fair policy as being parameterized by $\alpha$. Hence, according to this explanation, varying $\alpha$ not only controls the emphasis placed on fairness, but also changes the fairness measure itself. A question that arises is whether the $\alpha$-fair policy achieves the optimal efficiency-fairness tradeoff in practical resource allocation scenarios wherein the fairness measure does not depend on extrinsic parameters like $\alpha$.

To address this question, in this section we will investigate the relationship between the $\alpha$-fair policy and the optimal EJT. We begin by studying the case of $M=2$ users. The main result in this case is stated in the following proposition:

Proposition 1. Let $\mathcal{C}$ be an arbitrary set, possibly discrete, and let $M=2$. For any $\alpha \in(0, \infty)$, the $\alpha$-fair benefit vector $\mathbf{x}_{\alpha}^{\star}$ generated by (2) achieves the optimal EJT.

Proof: See Appendix A.

Proposition 1 shows that for an arbitrary set $\mathcal{C}$ and $M=2$, the $\alpha$-fair policy yields tradeoffs that are optimal from Jain's index perspective. However, this result does not necessarily carry over to cases with $M>2$ users. To show this, we constructed counter examples for $M=3$ and $M=4$. The case of $M=4$ yields deeper insight and will be explained in more detail.

Example 1. Let $\mathcal{C}$ contain two benefit vectors, i.e., $\mathcal{C}=\{\mathbf{x}, \mathbf{y}\}$, where $\mathbf{x}=[8,8,90,90]$ and $\mathbf{y}=[7,14,27,86]$.

For $\alpha=2$, maximizing the $\alpha$-fair utility yields $\mathbf{y}$ because $U_{2}(\mathbf{y})>U_{2}(\mathbf{x})$. However, $\eta(\mathbf{x})=196, \eta(\mathbf{y})=134, J(\mathbf{x})=$ 0.59 and $J(\mathbf{y})=0.54$, that is, $\eta(\mathbf{x})>\eta(\mathbf{y})$ and $J(\mathbf{x})>J(\mathbf{y})$, which implies that $\mathrm{x}$ is the optimal EJT benefit vector. This agrees with intuition since, by inspection, $\mathrm{x}$ offers $75 \%$ of the users higher benefits than $\mathbf{y}$.
Drawing more insight from the above example, we will show that the efficiency gap between the benefit vectors generated by the optimal EJT and those generated by the $\alpha$-fair one can be unbounded. To show that, let us modify $\mathcal{C}$ in the above example such that $\mathcal{C}=\{\mathbf{x}, \mathbf{y}, \hat{\mathbf{x}}, \hat{\mathbf{y}}\}$, where $\hat{\mathbf{x}}=c \mathbf{x}, \hat{\mathbf{y}}=c \mathbf{y}$, and $c>1$ is some constant. In this case, it can be easily verified that $\hat{\mathbf{y}}$ is the $\alpha$-fair benefit vector and $\hat{\mathbf{x}}$ is the optimal EJT benefit vector. Furthermore, because Jain's index is invariant under scaling, $J(\hat{\mathbf{x}})=J(\mathbf{x})>J(\hat{\mathbf{y}})=J(\mathbf{y})$. However, direct computation reveals that $\eta(\hat{\mathbf{x}})-\eta(\hat{\mathbf{y}})=c(\eta(\mathbf{x})-\eta(\mathbf{y}))$. Hence, an unbounded $c$, results in an unbounded difference in efficiency between the optimal EJT and the $\alpha$-fair benefit vectors. The existence of such $c$ depends, of course, on $\mathcal{C}$. In fact, it will be shown later that the structure of $\mathcal{C}$ is intimately related to the optimal EJT.

Another insight that can be drawn from the above example is that the $\alpha$-fair benefit vector corresponding to $\alpha=0$ is $\mathbf{x}$, which, from Jain's index perspective, is fairer than the $\alpha$ fair benefit vector corresponding to $\alpha=2$. This shows that, although increasing $\alpha$ results in benefit vectors that are fairer in the senses considered in [1] and [2], it does not necessarily improve fairness in the Jain's index sense.

Many applications, including wireless communications ones, involve the tradeoff between the benefit vectors of more than two users. Since in these cases, maximizing the $\alpha$-fair utility does not necessarily yield benefit vectors that achieve the optimal EJT (cf. Example 1), in the next section we will develop a technique for achieving this tradeoff.

\section{THE OPTIMAL EFFICIENCY-JAIN TRADEOFF POLICY}

In this section, we develop a generic technique for obtaining the optimal EJT for an arbitrary set $\mathcal{C}$. To enable practical implementation of this technique, we identify conditions, which, when satisfied by the set $\mathcal{C}$, renders the underlying optimization problems easy to solve. We will then provide instances in which these conditions are satisfied in practice. A geometric interpretation that commends the use of Jain's index as a fairness measure is then provided. We conclude this section by providing an alternate formulation that will prove useful in characterizing and achieving the optimal EJT in time-varying scenarios.

\section{A. A Technique for Obtaining the Optimal EJT for an Arbi- trary $\mathcal{C}$}

Let $\sigma$ be a threshold on the minimum efficiency, and let $\mathcal{X}_{\sigma}$ be the set of all benefit vectors that yield an efficiency greater than $\sigma$ and, at the same time, maximize Jain's index, that is,

$$
\mathcal{X}_{\sigma} \triangleq\left\{\mathbf{x} \mid \mathbf{x}=\arg \max _{\eta(\mathbf{x}) \geq \sigma, \mathbf{x} \in \mathcal{C}} J(\mathbf{x})\right\} .
$$

We note that the cardinality of $\mathcal{X}_{\sigma}$ depends on $\mathcal{C}$. Furthermore, some elements in $\mathcal{X}_{\sigma}$ may satisfy the condition $\eta(\mathbf{x}) \geq \sigma$ in (6) with a strict inequality. Since we are seeking the benefit vectors that achieve the optimal EJT, we pick those vectors in $\mathcal{X}_{\sigma}$ that maximize $\eta(\mathbf{x})$. In particular, let $\mathbf{x}_{\sigma}^{\star}$ be one of the benefit vectors that achieve the optimal EJT corresponding to $\sigma$, that is,

$$
\mathbf{x}_{\sigma}^{\star} \in \arg \max _{\mathbf{x} \in \mathcal{X}_{\sigma}} \eta(\mathbf{x}) .
$$


From (6) and (7), it can be seen that, for the given $\sigma, \mathbf{x}_{\sigma}^{\star}$ achieves the optimal EJT in Definition 2. Hence, the set of all EJT-optimal benefit vectors can be obtained by decrementing $\sigma$ from $\sigma_{\max }=\max _{\mathbf{x} \in \mathcal{C}} \eta(\mathbf{x})$ to $\sigma_{\min }=\min _{\mathbf{x} \in \mathcal{C}} \eta(\mathbf{x})$ in $K+1$ steps, each of size $\delta$. For each step $k$, the optimization problems in (6) and (7) corresponding to $\sigma=\sigma_{\max }-k \delta$ are solved, $k=0, \ldots, K$; a smaller $\delta$ results in evaluating more points and therefore obtaining a smoother EJT curve. This policy is presented formally in Procedure 1. Inspection of this procedure

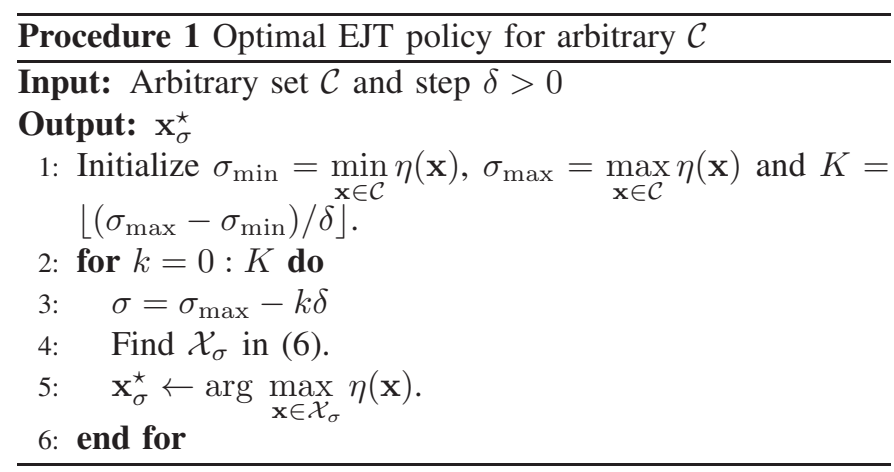

reveals that the main difficulty in obtaining $\mathbf{x}_{\sigma}^{\star}$ lies in finding a solution of the optimization problem in (6), let alone finding the entire set $\mathcal{X}_{\sigma}$. This difficulty arises because $J(\mathbf{x})$ is a nonconcave function, even when $\mathcal{C}$ is a convex set. If the dimension of the set of feasible benefits is large, Procedure 1 can be prohibitively complex to implement in real-time scenarios. In such cases, this procedure might be used as a benchmark for less costly algorithms that approximate the solution of the underlying non-convex optimization problems. The accuracy of such algorithms depends on the approximation technique and the properties of $\mathcal{C}$. The complexity of Procedure 1 motivates us to seek conditions, which, when satisfied by $\mathcal{C}$, the optimal EJT can be readily obtained.

\section{B. A Property for Ensuring Tractability}

In order to render the optimization problems underlying (6) easy to solve, we begin by identifying a class of sets $\mathcal{C}$ which satisfy what we refer to as the "monotonic tradeoff property". To do so, let $J_{\sigma}^{\star}$ denote the maximum Jain's index corresponding to an efficiency $\eta(\mathbf{x})=\sigma$, i.e.,

$$
J_{\sigma}^{\star}=\max _{\eta(\mathbf{x})=\sigma, \mathbf{x} \in \mathcal{C}} J(\mathbf{x}) .
$$

By definition, $J_{\sigma}^{\star}$ is unique. However, it might be achieved by multiple benefit vectors.

Using (8), we are now ready to define the monotonic tradeoff property.

Definition 3 (Monotonic Tradeoff Property). A set $\mathcal{C}$ is said to possess the monotonic tradeoff property if $J_{\sigma}^{\star}$ is strictly decreasing in $\sigma$, for $\sigma \geq \sigma^{\star}$, and constant otherwise.

This definition states that a set that possesses the monotonic tradeoff property is one in which any decrease in efficiency results in a strict increase in the Jain's index, until $\sigma^{\star}$ is reached. Decreasing efficiency beyond $\sigma^{\star}$ maintains Jain's index at its maximum. In other words,

$$
J_{\sigma^{\star}}^{\star}=\max _{\eta(\mathbf{x})=\sigma^{\star}, \mathbf{x} \in \mathcal{C}} J(\mathbf{x})=\max _{\mathbf{x} \in \mathcal{C}} J(\mathbf{x}) .
$$

An instance in which $\mathcal{C}$ satisfies the monotonic tradeoff property is shown in Fig. 1(a) and the corresponding EJT is shown in Fig. 1(b). These figures will be discussed in the next section.

We will now show how the monotonic tradeoff property facilitates finding the benefit vectors that achieve the optimal EJT. When this property is satisfied, the inequality $\eta(\mathbf{x}) \geq \sigma$ in (6) is satisfied with equality when $\sigma>\sigma^{\star}$ because $J_{\sigma}^{\star}$ is strictly decreasing in $\sigma$. In this case, the optimization in (6) is equivalent to that in (8). We now use (8) to obtain an equivalent convex formulation. By definition, $J(\mathbf{x})=\frac{\eta^{2}(\mathbf{x})}{M\|\mathbf{x}\|^{2}}$. Hence, when $\eta(\mathbf{x})=\sigma$, the objective in (8) can be expressed as $\frac{\sigma^{2}}{M\|\mathbf{x}\|^{2}}$ and (8) can be cast in the following equivalent form:

$$
\min _{\eta(\mathbf{x})=\sigma, \mathbf{x} \in \mathcal{C}}\|\mathbf{x}\|^{2} .
$$

In contrast with (6), the objective in (10) is convex. In fact, this objective is strictly convex, which implies that when $\mathcal{C}$ too is convex, the optimization problem in (10) is easy to solve and its solution is unique [14, p. 397]. In addition, if $\mathcal{C}$ is not convex and (10) has multiple solutions, all these solutions will achieve the same EJT as they all have the same efficiency, $\sigma$, and the same Jain's index. This eliminates the requirement for finding all solutions in (6) since any solution of (10) achieves the optimal EJT. To summarize, if the monotonic tradeoff property in Definition 3 is satisfied, $\mathbf{x}_{\sigma}^{\star}$ can be found by solving (10), which is significantly easier than solving the optimization problems in (6) and (7) for an arbitrary $\mathcal{C}$.

Similar to Procedure 1, the benefit vectors that achieve the optimal EJT can be obtained by varying $\sigma$ from $\sigma_{\max }$ to $\sigma_{\min }$. However, when $\mathcal{C}$ possesses the monotonic tradeoff property, we can find $\mathbf{x}_{\sigma}^{\star}$ by solving (10) for each $\sigma$. This policy is presented formally in Procedure 2 below.

Procedure 2 Optimal EJT for $\mathcal{C}$ possessing the monotonic tradeoff property

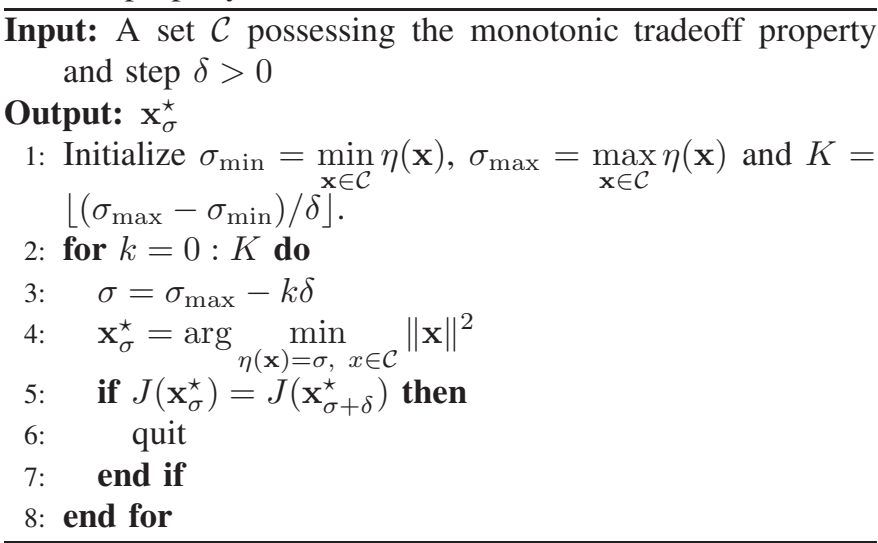

\section{Sufficient Conditions for Satisfying the Monotonic Tradeoff Property}

In the previous section, we have shown that finding the set of benefit vectors that achieve the optimal EJT is significantly 
simplified when the set $\mathcal{C}$ possesses the monotonic tradeoff property. Unfortunately, we have not been able to identify a distinguishing feature that is necessary for a set to possess the monotonic tradeoff property. For instance, this property can be possessed by sets that are either continuous or discrete, convex or otherwise. This observation suggests that deriving necessary conditions might be elusive. However, we have been able to obtain sufficient conditions, which, when satisfied, ensure that a given set possesses this property. Such conditions are provided in Theorem 1 below ${ }^{1}$.

Theorem 1. The set $\mathcal{C}$ possesses the monotonic tradeoff property if:

i. $\mathcal{C}$ is convex;

ii. $x_{\min } \mathbf{1}_{M} \in \mathcal{C}$; and

iii. every $\mathbf{x} \in \mathcal{C}$ satisfies $\mathbf{x} \succeq x_{\min } \mathbf{1}_{M}$, where $x_{\min } \geq 0$ provides a guarantee on the minimum benefit that each user receives.

Proof: See Appendix B.

To provide a graphical illustration of Theorem 1, in Fig. 1(a) we show a feasible set $\mathcal{C}$ satisfying the conditions of the theorem with $x_{\min }=0$ for a case with $M=2$ users. The EJT corresponding to the set in Fig. 1(a) is shown in Fig. 1(b).

To show how Fig. 1(b) is obtained, we begin by noting that, in Fig 1(a), the maximum Jain's fairness line $x_{1}=x_{2}$ passes through $\mathcal{C}$ and yields $J(\mathbf{x})=1$. The regular-weight dashed lines in this figure represent the constant efficiency levels, $\eta(\mathbf{x})=\sigma$, at different values of $\sigma$. For $\sigma \leq 5.33$, the points at which the dashed lines intersect the $x_{1}=x_{2}$ line lie inside $\mathcal{C}$. In this case, the maximal Jain's index, $J_{\sigma}^{\star}=1$. For $\sigma>5.33$, the dashed lines representing the $\eta(\mathbf{x})=\sigma$ levels intersect the $x_{1}=x_{2}$ line at points outside $\mathcal{C}$. For these efficiency levels, the maximal Jain's indices are strictly less than 1 and correspond to the points at which the dashed lines intersect with the boundary of $\mathcal{C}$. The optimal EJT benefit vectors are shown by the thick dashed line on the boundary of $\mathcal{C}$. The variation of $J_{\sigma}^{\star}$ with $\sigma$ is depicted in Fig. 1(b).

It can be seen from this figure that, in agreement with Theorem 1, the set $\mathcal{C}$ satisfies the monotonic tradeoff property in Definition 3 with $\sigma^{\star}=5.33$. In this figure, the optimal EJT corresponding to the thick dashed line on the boundary of $\mathcal{C}$ in Fig. 1(a) is represented by the thick dashed line to the right of $\sigma^{\star}$.

Although necessary conditions are not available, the sufficient conditions provided in Theorem 1 are relatively sharp. To illustrate that, we consider the optimal EJT for the $\operatorname{set} \mathcal{C}$ shown in Fig. 2(a). This set satisfies the first condition of Theorem 1, but does not satisfy the second and third conditions. In other words, $\mathcal{C}$ is convex, but there is no $x_{\min }$ such that $x_{\min } \mathbf{1}_{M} \in \mathcal{C}$ and $x \succeq x_{\min } \mathbf{1}_{M}, \forall \mathbf{x} \in \mathcal{C}$. We will now demonstrate that this set does not possess the monotonic tradeoff property in Definition 3.

For the set shown in Fig. 2(a), the maximum Jain's fairness line $x_{1}=x_{2}$ intersects $\mathcal{C}$ at one point, viz., $x_{1}=x_{2}=6$. At this point, the efficiency, $\sigma=12$ and Jain's index, $J(\mathbf{x})=1$.

\footnotetext{
${ }^{1}$ This theorem is a generalized version of the one we provided in [15] wherein $x_{\min }$ was restricted to be zero.
}

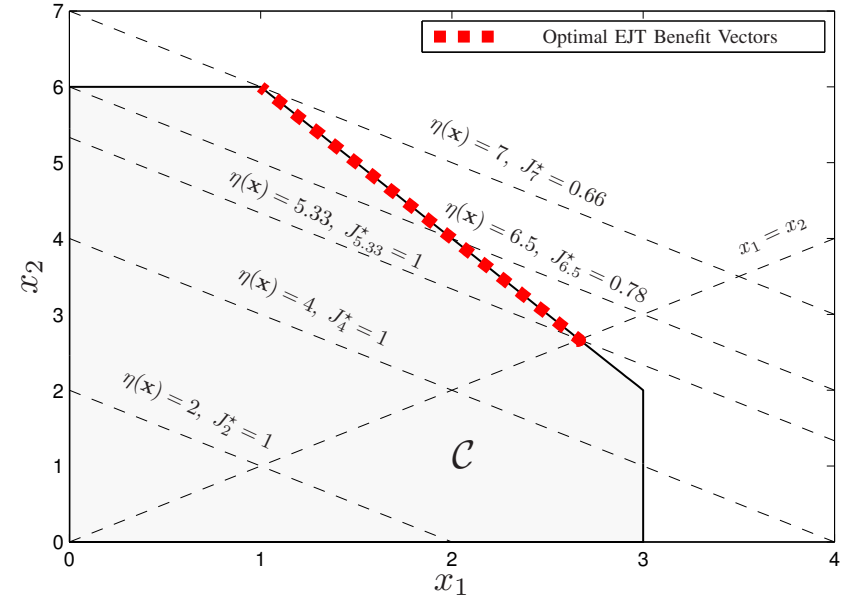

(a)

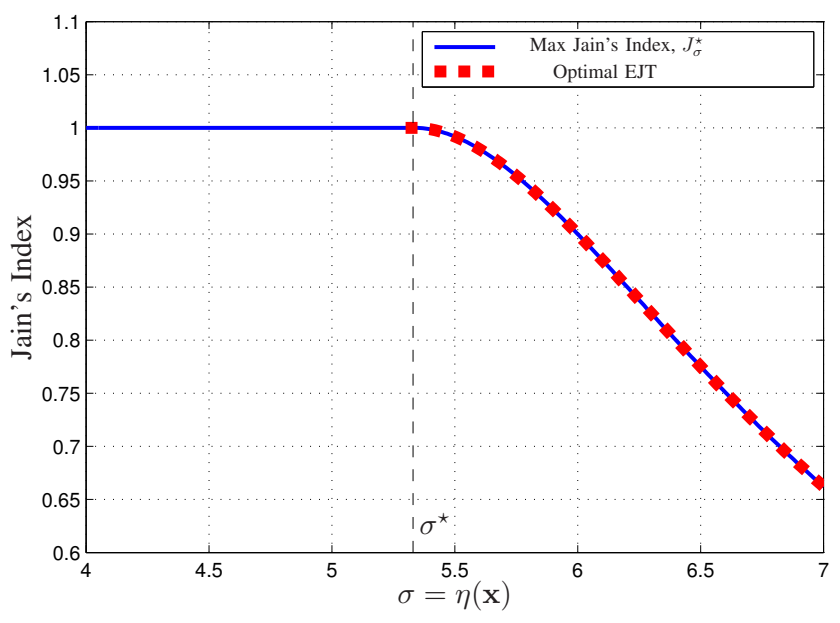

(b)

Fig. 1. (a) A convex set that satisfies the conditions in Theorem 1 and (b) its EJT curve.

At any other point in $\mathcal{C}$, Jain's index is strictly less than 1. To see why this implies that $\mathcal{C}$ does not possess the monotonic tradeoff property, we note that, for each dashed line representing constant $\sigma \in[3.6,12)$ and $\sigma \in(12,13]$ the maximal Jain's index, $J_{\sigma}^{\star}$, corresponds to the intersection of the dashed line with the non-vertical part of the boundary of $\mathcal{C}$. For $\sigma \in[3.6,12), J_{\sigma}^{\star}$ is strictly monotonically increasing in $\sigma$, and for $\sigma \in(12,13], J_{\sigma}^{\star}$, is strictly monotonically decreasing in $\sigma^{2}$ Hence, it can be seen that, for $\sigma<12$, the tradeoff is not meaningful, since, in that region, both $\eta(\mathbf{x})$ and $J_{\sigma}^{\star}(\mathbf{x})$ can be increased at the same time. The optimal EJT benefit vectors are shown by the thick dashed line on the boundary of $\mathcal{C}$.

The variation of $J_{\sigma}^{\star}$ with $\sigma$ is depicted in Fig. 2(b). As we pointed out, $J_{\sigma}^{\star}$ is strictly increasing for $\sigma<12$, implying that $\mathcal{C}$ does not satisfy the monotonic tradeoff property in Definition 3. For $\sigma \in(12,13]$, the optimal EJT corresponding to the thick dashed line on the boundary of $\mathcal{C}$ in Fig. 2(a) is represented by the thick dashed line to the right of $\sigma^{\star}$.

\footnotetext{
${ }^{2}$ For this $\mathcal{C}$, any $\sigma<3.6$ or $\sigma>13$ is not feasible.
} 


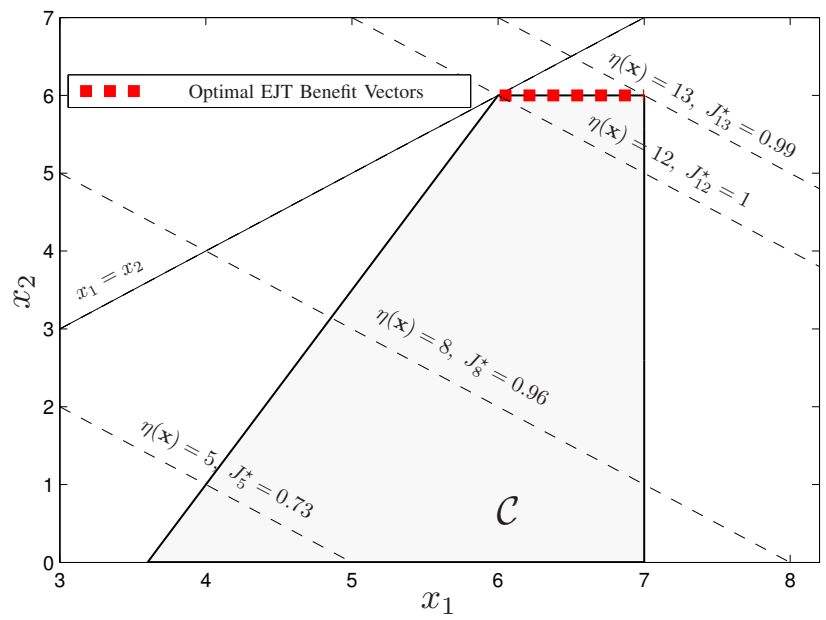

(a)

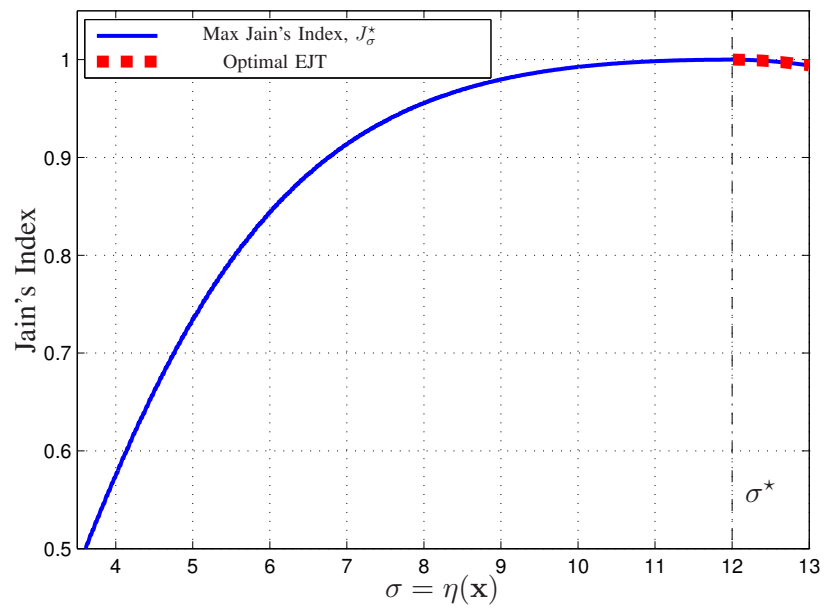

(b)

Fig. 2. (a) A convex set that does not satisfy the conditions in Theorem 1 and (b) its EJT curve.

\section{Practical Applications of Theorem 1}

The sufficient conditions given in Theorem 1 are quite general and can be applied to scenarios beyond those considered hereinafter. Indeed, these conditions are applicable, not only to communication systems, but also to other fields including social and economics ones. The conditions in Theorem 1 are naturally satisfied in various resource allocation problems in communication networks. For instance, in congestion control in elastic traffic communication networks [1], [10] the users share finite-capacity links and the goal is to assign the benefit vector $\mathbf{x}$, which represents the users' rates, in an efficient and fair manner. The set of feasible rates in this case is given by $\mathcal{C}=\left\{\mathbf{x} \mid \mathbf{A} \mathbf{x} \preceq \mathbf{c}, \mathbf{0}_{M} \preceq \mathbf{x}\right\}$, where the $\ell$-th entry of $\mathbf{c} \in \mathbb{R}_{+}^{\mathcal{L}}$ is the capacity of link $\ell, \ell=1, \ldots, \mathcal{L}$, and $\mathbf{A}$ is a matrix with binary entries that represent the assignment of users to links. In this case, the set $\mathcal{C}$ is a convex polyhedron [14, $\mathrm{p}$. 31] containing $\mathbf{0}_{M}$, and thereby satisfying the conditions of Theorem 1 with $x_{\min }=0$. Hence, $\mathcal{C}$ satisfies the monotonic tradeoff property and Procedure 2 can be used to find all the optimal EJT rate vectors.

Another example is the allocation of radio resources in the downlink of cellular networks, which will be discussed in Section VI in more detail.

\section{E. Geometric Interpretation of the Optimal EJT}

When $\mathcal{C}$ satisfies the sufficient conditions given in Theorem 1, optimal EJT benefit vectors $\left\{\mathbf{x}_{\sigma}^{\star}\right\}$ have an interesting geometric interpretation. To see that, we use (10) to write

$$
\begin{aligned}
\mathbf{x}_{\sigma}^{\star} & =\arg \min _{\eta(\mathbf{x})=\sigma, \mathbf{x} \in \mathcal{C}} \sum_{m=1}^{M} x_{m}^{2} \\
& =\arg \min _{\eta(\mathbf{x})=\sigma, x \in \mathcal{C}} \sum_{m=1}^{M}\left(x_{m}^{2}-2 \frac{\sigma}{M} \eta(\mathbf{x})+\frac{\sigma^{2}}{M^{2}}\right) \\
& =\arg \min _{\eta(\mathbf{x})=\sigma, x \in \mathcal{C}}\left\|\mathbf{x}-\frac{\sigma}{M} \mathbf{1}_{M}\right\|^{2} .
\end{aligned}
$$

The last equality states that $\mathbf{x}_{\sigma}^{\star}$ is the unique Euclidean projection [14, p. 397] of the equal allocation vector $\frac{\sigma}{M} \mathbf{1}_{M}$ onto the set $\{\mathbf{x} \mid \eta(\mathbf{x})=\sigma, \mathbf{x} \in \mathcal{C}\}$. In other words, a benefit vector $\mathbf{x}_{\sigma}^{\star}$ achieves the optimal EJT if there is no feasible benefit vector $\mathbf{y} \neq \mathbf{x}_{\sigma}^{\star}$ such that $\eta(\mathbf{y})=\sigma$ is closer to the fairest solution $\frac{\sigma}{M} \mathbf{1}_{M}$. This interpretation commends the use of Jain's index as a fairness measure and is illustrated in Fig. 3. It also complements the interpretation given in [16] that Jain's index represents the angular deviation from a scaled all-one vector.

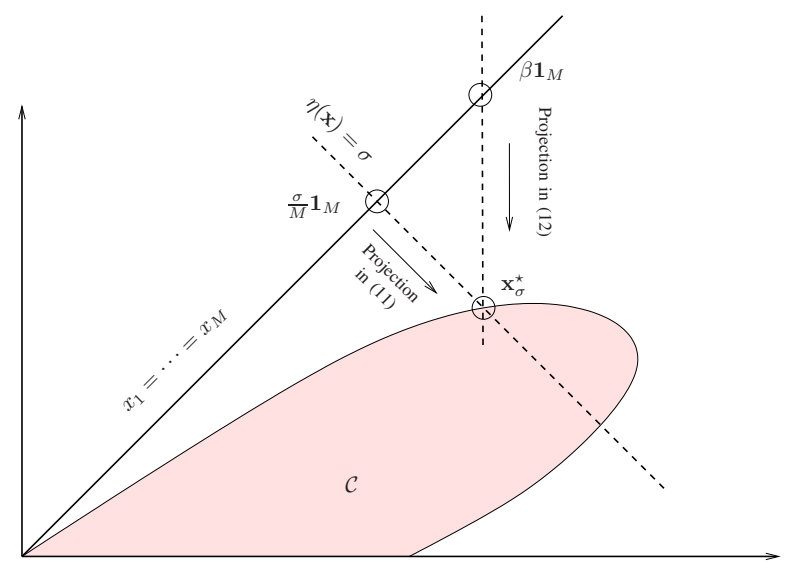

Fig. 3. The optimal EJT benefit vector, $\mathbf{x}_{\sigma}^{\star}$, is the unique projection of the fairest vector $\frac{\sigma}{M} \mathbf{1}_{M}$ onto the set $\{\mathbf{x} \mid \eta(\mathbf{x}) \stackrel{\sigma}{=} \sigma, \mathbf{x} \in \mathcal{C}\}$. Projection of $\beta \mathbf{1}_{M}$ onto $\mathcal{C}$ is also shown.

\section{F. An Alternate Formulation}

In Section IV-B it was shown that, if the set $\mathcal{C}$ possesses the monotonic tradeoff property, the optimal EJT benefit vectors can be obtained by solving the optimization problem in (10) for each $\sigma$. As such, the solution of (10) can be viewed as being parameterized by $\sigma$.

Although the form in (10) is convenient for providing an explicit characterization of the optimal EJT, the equality constraint therein renders it difficult to utilize in some applications. An instance of these applications is considered in the next section, wherein the instantaneous allocations of radio 
resources are to be updated for optimizing long-term average rates.

One approach to address the aforementioned difficulty is to incorporate the equality constraint into the objective by eliminating one of the variables [14, pp. 523-524]. However, this approach can be shown to result in complicating the computation of gradient vectors necessary for the development of effective procedures for updating resource allocations. To alleviate this difficulty, we now provide an alternate formulation for (10). In this formulation, the efficiency $\sigma$ is implicitly accounted for by a non-negative parameter $\beta$ in the objective, and the benefit vectors are only constrained to lie in $\mathcal{C}$. In particular, when the conditions of Theorem 1 are satisfied, we have that for any $\sigma=\eta(\mathbf{x})$, the formulation in (10) is equivalent to

$$
\min _{\mathbf{x} \in \mathcal{C}}\|\mathbf{x}\|^{2}-2 \beta \eta(\mathbf{x}),
$$

for some $\beta \in[0, \infty)$. To see this, we let $\sigma$ be the efficiency corresponding to the solution of (12) for a given $\beta \in[0, \infty)$; letting $\beta=0$ corresponds to $\sigma_{\min }$, the minimum feasible efficiency, and letting $\beta \rightarrow \infty$ corresponds to $\sigma \rightarrow \sigma_{\max }$, the maximum feasible efficiency. For intermediate values of $\beta$, it can be shown that the efficiency generated by (12) is monotonically increasing in $\beta$. Thus, the EJT obtained by letting $\beta$ span the interval $[0, \infty)$ in (12) is the same as that obtained by letting $\sigma$ span the interval $\left[\sigma_{\min }, \sigma_{\max }\right)$ in (10).

Similar to the observation made in the previous section, the objective in (12) can be equivalently expressed as $\left\|\mathbf{x}-\beta \mathbf{1}_{\mathbf{M}}\right\|^{2}$. Hence, the optimum EJT benefit vector generated by (12) is the Euclidean projection of the benefit vector $\beta \mathbf{1}_{\mathbf{M}}$ onto the feasible set $\mathcal{C}$. A subtle difference between the objectives in (12) and (11) is that, the projection in (12) is onto $\mathcal{C}$, whereas that in (11) is onto the intersection of $\mathcal{C}$ with the hyperplane $\eta(\mathbf{x})=\sigma$; cf. Fig. 3 .

To obtain further insight into the role of $\beta$, we note that, because of the monotonic tradeoff property, the solution of (10) remains unchanged if the equality constraint is replaced by the inequality $\eta(\mathbf{x}) \geq \sigma$. Hence, $2 \beta$ can be regarded as the Lagrange multiplier corresponding to this constraint and is, therefore, non-negative.

\section{IMPlications of THEOREM 1 IN RAdio ResourCE AllocAtions}

In this section, we consider the scheduling of radio resources to multiple users in the downlink of a wireless communication network using the multiple access technique based on orthogonal frequency division multiplexing (OFDM). Resources are divided into $N$ (time-frequency) resource blocks (RBs) [17], and the goal is to allocate these RBs to $M$ users in a way that is both "efficient and fair". We consider quasi-static and ergodic time-varying channels. For quasistatic channels, we consider scheduling with and without timesharing. In the case of time-sharing, the scheduling variables are continuous and the corresponding set of feasible benefit vectors, $\mathcal{C}$, satisfies the conditions of Theorem 1 . In contrast, in the case without time-sharing, the scheduling variables are discrete and $\mathcal{C}$ does not satisfy the conditions of Theorem 1 . For ergodic time-varying channels, time-sharing is not plausible and the scheduling variables are discrete. In spite of that, the corresponding set of feasible benefit vectors, $\mathcal{C}$, can be shown to satisfy the conditions of Theorem 1. It is worth noting that several communication scenarios are neither quasi-static nor ergodic time-varying, e.g., non-ergodic communication scenarios [18]. For these scenarios, an appropriate efficiency measure would account for the probability of outage.

\section{A. Case 1: Quasi-Static Channels}

Under quasi-static channel conditions and given modulation and coding schemes, the data rate of each user $m \in \mathcal{M} \triangleq$ $\{1, \ldots, M\}$ on $\mathrm{RB} n \in \mathcal{N} \triangleq\{1, \ldots, N\}$, which we denote by $r_{m n}$, is a deterministic quantity known to the transmitter. The transmitter objective is to determine a fair RB allocation that ensures efficient communication of data to the users. To achieve this goal, let $\rho_{m n} \in[0,1]$ be a scheduling variable that assigns RB $n$ to user $m$ for a fraction $\rho_{m n}$ of the signalling interval [19]. At each time instant, each RB is used by at most one user, and thus $\sum_{m=1}^{M} \rho_{m n} \leq 1$. The total data rate (benefit) of user $m$ is given by $x_{m}=\sum_{n=1}^{N} \rho_{m n} r_{m n}$ and the efficiency of the network is given by the total sum-rate, which is given by $\eta(\mathbf{x})=\sum_{m=1}^{M} x_{m}$. The set of achievable rates (benefits) of the users is given by

$$
\begin{gathered}
\mathcal{C}=\left\{\mathbf{x} \mid x_{m}=\sum_{n=1}^{N} \rho_{m n} r_{m n}, \sum_{m=1}^{M} \rho_{m n} \leq 1, \rho_{m n} \in[0,1],\right. \\
\left.x_{m} \geq x_{\min }, m=\in \mathcal{M}, n \in \mathcal{N}\right\}
\end{gathered}
$$

where $x_{\min } \geq 0$ represents a feasible threshold on the minimum rate that must be delivered to each user. Using this description, the goal of the transmitter can be cast as to determine the set $\left\{\rho_{m n}\right\}$ that results in rate vectors $\mathbf{x}$ that span the optimal EJT. This goal can be achieved by invoking the results of Theorem 1 . In particular, we note that the set $\mathcal{C}$ in (13) is convex and contains the vector $x_{\min } \mathbf{1}_{M}$. Hence, the conditions of Theorem 1 are satisfied and $\mathcal{C}$ possesses the monotonic tradeoff property. Based on this observation, Procedure 2 will be used in Section VI-A to obtain $\left\{\rho_{m n}\right\}$ that achieve every point on the optimal EJT.

When the RBs are not time-shared, $\left\{\rho_{m n}\right\}$ assume binary values (i.e., $\rho_{m n} \in\{0,1\}$ ), resulting in the set $\mathcal{C}$ being nonconvex. In this case, $\mathcal{C}$ may not possess the monotonic tradeoff property and Procedure 1 can be used to obtain the optimal $\left\{\rho_{m n}\right\}$. However, we note that, in contrast with Procedure 2, which is used when time-sharing is allowed and $\left\{\rho_{m n}\right\}$ are continuous, Procedure 1 is significantly more computationallydemanding.

\section{B. Case 2: Ergodic Time-Varying Channel}

We now consider the problem of determining the radio resource allocations that span the optimal EJT when the channels are ergodic and time-varying. Before providing the mathematical framework for this case, we begin by noting that, from a practical perspective, one is typically interested in average, rather than instantaneous, rates [17], [18]. In those cases, one might be tempted to apply the same approach in 
the previous section on instantaneous realizations of the channels. Although this would guarantee optimal tradeoff between efficiency and fairness in every time instant, it suffers from a major drawback that was alluded to in [17]. In particular, applying that instantaneous strategy does not necessarily lead to long-term average rates that are optimal from an EJT perspective.

For ergodic time-varying channels considered in this section, the channel gains assume random values in every time slot $t$. When these gains are available at the transmitter and the receivers, the instantaneous data rate of each user $m \in \mathcal{M}$ on each RB $n \in \mathcal{N}$ is denoted by $r_{m n}(t)$. To achieve various points on the long-term average optimal EJT, we define, similar to the previous section, scheduling variables. However, in the current case of time-varying channels, these variables are binary, indexed by $t$, and denoted by $\left\{\rho_{m n}(t)\right\}$. The reason that $\left\{\rho_{m n}(t)\right\}$ are assumed to be binary is that the channel gains take on different values in each time slot, rendering time-sharing implausible. In other words, for each channel realization, the role of $\left\{\rho_{m n}(t)\right\}$ is to assign each $\mathrm{RB}$ to a particular user. Updating $\left\{\rho_{m n}(t)\right\}$ to achieve points on the long-term average optimal EJT will be accomplished using the gradient scheduling algorithm, which we describe next.

1) The Gradient Scheduling Algorithm: The gradient scheduling algorithm is a particular instance of adaptive algorithms that enable efficient solving of stochastic optimization problems wherein the utilities to be maximized involve long-term averaging over an ergodic process; see e.g., [17], [20]. The key idea that underlies such an algorithm is to use gradient-based steps to update the optimization variables sequentially using current and previous observations of the process. In addition to its relative simplicity, variants of the gradient scheduling algorithm were shown in [17] and [20] to yield the optimal solution of the stochastic optimization problem as the number of observations becomes sufficiently large.

To apply this algorithm to the current framework, let $R_{m}(t)$ be the data rate scheduled to user $m$ at time $t$, i.e., $R_{m}(t)=\sum_{n=1}^{N} \rho_{m n}(t) r_{m n}(t)$. Maximizing the standard average rate of user $m$ directly results in spurious behaviour [21], which can be alleviated by using the exponentially-weighted moving average instead. To do so, let $\mu \in(0,1)$ be a small positive scalar [20] and define $W_{m}(t)$ to be

$$
\begin{aligned}
W_{m}(t) & =\mu \sum_{i=0}^{t}(1-\mu)^{i-t} R_{m}(i) \\
& =(1-\mu) W_{m}(t-1)+\mu R_{m}(t) .
\end{aligned}
$$

For notational convenience, let $\mathbf{W}(t)=\left[W_{1}(t), \ldots, W_{M}(t)\right]^{T}$ and $\mathbf{R}(t)=\left[R_{1}(t), \ldots, R_{M}(t)\right]^{T}$. Since our goal is optimize long-term (i.e., steady-state) average rates, the benefit vector of the $M$ users, $\mathbf{x}$, can be defined to be $\lim _{t \rightarrow \infty} \mathbf{W}(t)$. Using the above notation, the idea behind gradient scheduling algorithm can be described as follows: Given the exponentially-weighted average rates at $t-1, \mathbf{W}(t-1)$, and the instantaneous rates, $\left\{r_{m n}(t)\right\}$, the task of the scheduler is to determine the instantaneous scheduling variables, $\left\{\rho_{m n}(t)\right\}$, in such a way that maximizes a given system utility $U(\mathbf{x}): \mathbb{R}^{M} \rightarrow \mathbb{R}$. We will later show how $U(\cdot)$ can be chosen to account for various tradeoff criteria. Since at time $t$ the scheduler knows the previous values of $\mathbf{W}(t)$, but not future ones, its instantaneous decisions, $\left\{\rho_{m n}(t)\right\}$, can only depend on $\mathbf{W}(t-1)$ and $\left\{r_{m n}(t)\right\}$. In the gradient scheduling algorithm, the scheduler generates these decisions using the first order Taylor's series expansion of $U(\mathbf{W}(t))$ around $\mathbf{W}(t-1)$. In particular, using (14) with a sufficiently small $\mu$, we can write $U(\mathbf{W}(t)) \approx U(\mathbf{W}(t-1))+\mu \nabla U(\mathbf{W}(t-1))^{T}(\mathbf{R}(t)-\mathbf{W}(t-$ $1))$. Noting that, at time $t, U(\mathbf{W}(t-1))$ is constant, it can be seen that only the term containing $\mathbf{R}(t)$ depends on $\left\{\rho_{m n}(t)\right\}$. Hence, maximizing $U(\mathbf{W}(t))$ is approximately equivalent to solving

$$
\max _{\left\{\rho_{m n}(t)\right\} \in \mathcal{S}} \nabla U(\mathbf{W}(t-1))^{T} \mathbf{R}(t),
$$

where $\mathcal{S} \triangleq\left\{\left\{\rho_{m n}\right\} \mid \sum_{m=1}^{M} \rho_{m n} \leq 1, \rho_{m n} \in\{0,1\}, \forall m \in\right.$ $\mathcal{M}, n \in \mathcal{N}\}$.

Invoking the definition of $\mathbf{R}(t)$, the solution of (15) can be expressed as

$$
\rho_{m n}(t)= \begin{cases}1, & \text { if } m=\arg \max _{m \in \mathcal{M}} \frac{\partial U(\mathbf{W}(t-1))}{\partial W_{m}(t-1)} r_{m n}(t) \\ 0, & \text { otherwise. }\end{cases}
$$

It is shown in [17], [20], [21] that, when the rate processes $\left\{r_{m n}(t)\right\}$ are ergodic and the utility $U(\cdot)$ is concave, the scheduling variables obtained by the gradient scheduling algorithm in (16) yield a long-term average rate vector $\mathbf{x}$ that maximizes $U(\mathbf{x})$, asymptotically as $\mu \rightarrow 0$.

2) Application of Gradient Scheduling to EfficiencyFairness Utilities: With a proper choice of $U(\cdot)$ in (16), the gradient scheduling algorithm can be made to yield instantaneous schedules that attain long-term optimal efficiencyfairness tradeoffs for various fairness measures. To show this, we consider the case in which the set $\mathcal{C}$ contains the longterm average rate benefit vectors corresponding to all possible choices of the scheduling variables $\left\{\rho_{m n}(t)\right\}$, i.e.,

$$
\begin{gathered}
\mathcal{C}=\bigcup_{\left\{\rho_{m n}(t)\right\} \in \mathcal{S}, \forall t}\left\{\mathbf{x} \mid x_{m}=\lim _{t \rightarrow \infty} \mu \sum_{i=0}^{t}(1-\mu)^{i-t} R_{m}(i),\right. \\
\left.R_{m}(i)=\sum_{n=1}^{N} \rho_{m n}(i) r_{m n}(i)\right\} .
\end{gathered}
$$

This definition implies that the instantaneous scheduling variables generated by (16) yield long-term average rate benefit vectors that lie in $\mathcal{C}$.

a) Achieving $\alpha$-Fairness: When the fairness measure is given by the $\alpha$-fair utility, the function $U(\cdot)$ in (16) is replaced with the utility $U_{\alpha}(\mathbf{x})$ in (3). Since this utility is concave for all $\alpha \in[0, \infty)$, the gradient scheduling algorithm with $\mu \rightarrow 0$ can be used to obtain the instantaneous schedules that yield the corresponding long-term optimal average rate vectors. In this case, these schedules are given by

$$
\rho_{m n}(t)= \begin{cases}1, & \text { if } m=\arg \max _{m \in \mathcal{M}}\left(W_{m}(t-1)\right)^{-\alpha} r_{m n}(t), \\ 0, & \text { otherwise. }\end{cases}
$$


b) Achieving Optimum EJT: We now show how to use the gradient scheduling algorithm to obtain optimum EJT. For simplicity we restrict our attention to the case of $x_{\min }=0$. To consider this case, we note that, with $x_{\min }=0$, the set of feasible benefit vectors defined in (17) satisfies the conditions of Theorem 1. In particular, this set is convex and contains the all-zero vector $\mathbf{0}_{M}$. To see that $\mathcal{C}$ is convex in $\mathbf{x}$, we note that, for any two long-term average rate benefit vectors $\mathbf{x}_{1}, \mathbf{x}_{2} \in \mathcal{C}$ and any $\theta \in[0,1]$, the line segment $\theta \mathbf{x}_{1}+(1-\theta) \mathbf{x}_{2}$ is also in $\mathcal{C}$ [17]. That $\mathbf{0}_{M} \in \mathcal{C}$ follows from the fact that setting the scheduling variables $\rho_{m n}(t)=0$ for all $m, n$ and $t$ is feasible, i.e., the all-zero $M \times N$ matrix $\mathbf{0}_{M N} \in \mathcal{S}$.

Now that the conditions of Theorem 1 are satisfied, we know that $\mathcal{C}$ possesses the monotonic tradeoff property in Definition 3, and Procedure 2 can be used to find the optimum EJT long-term average rates. In this procedure, the optimization problem in (10) is solved for various choices of $\sigma$. For each value of $\sigma$, the problem in (10) involves a constraint on the sum of the long-term average rates. Unfortunately, incorporating such a constraint in the gradient scheduling algorithm is not straightforward and hence, this algorithm cannot be used directly to solve (10) in the current stochastic framework. To circumvent this difficulty, we use the alternate formulation of (10) given in (12). Using that formulation, the gradient scheduling algorithm can be applied with the utility $U(\cdot)$ in (16) replaced with

$$
U_{\beta}(\mathbf{x})=-\|\mathbf{x}\|^{2}+2 \beta \eta(\mathbf{x}) .
$$

where, as explained in Section IV-F, $\beta \in[0, \infty)$.

Using the utility in (19), the instantaneous schedules that yield the optimal EJT long-term average rate vectors, for a given $\beta$, are given by

$\rho_{m n}(t)= \begin{cases}1, & \text { if } m=\arg \max _{m \in \mathcal{M}}\left(\beta-W_{m}(t-1)\right) r_{m n}(t), \\ 0, & \text { otherwise. }\end{cases}$

Now that we have shown how the gradient scheduling algorithm can be used to yield optimal EJT long-term average rate vectors, in the next section we will investigate the performance of this algorithm in practical wireless communication scenarios.

\section{Numerical And Simulation Results}

In this section, we compare the EJT achieved by the optimal and the $\alpha$-fair based schedulers for two cases. In the first case, the channels between the base station (BS) and the users are quasi-static and in the second case these channels are ergodic time-varying.

\section{A. Case 1: Quasi-Static Channels}

We consider one realization of a quasi-static network with $M=4$ users and $N=5$ RBs. As an example, we assume that the rate matrix $\mathbf{r}=\left[r_{m n}\right]$ is given by

$$
\mathbf{r}=\left[\begin{array}{ccccc}
544 & 648 & 807 & 544 & 722 \\
388 & 92 & 223 & 388 & 56 \\
35 & 544 & 35 & 722 & 56 \\
35 & 56 & 35 & 92 & 35
\end{array}\right]
$$

The rates in this matrix are given in $\mathrm{Kb} / \mathrm{s}$ and were obtained from simulating a practical scenario based on the Long Term Evolution (LTE) standard [22]. In the considered scenario, users 1 and 2 are closer to the BS than users 3 and 4, and the wireless channels are quasi-static frequency-flat and Rayleigh fading on each RB.

The comparisons between the optimal EJT and the tradeoff achieved by the $\alpha$-fair policy for the case when the RBs can be time-shared among users are shown in Fig. 4(a) and Fig. 4(b). In Fig. 4(a), it is assumed that there is no minimum rate guarantee, i.e., $x_{\min }=0$, while in Fig. 4 (b) the minimum rate guarantee is assumed to be $50 \mathrm{~Kb} / \mathrm{s}$, i.e., $x_{\min }=50 \mathrm{~Kb} / \mathrm{s}$. Procedure 2 was used to obtain the optimal EJT, and the tradeoff achieved by the $\alpha$-fair policy was obtained by solving (2). The convex optimization problems underlying Procedure 2 and the $\alpha$-fair policy were solved using 'fmincon', which is available in the MATLAB optimization toolbox. From both figures, it can be seen that, while these tradeoffs are close to each other for small and large values of $\alpha$, for intermediate values, the tradeoff generated by the optimal EJT policy is significantly better than that generated by the $\alpha$-fair one. For example, for a Jain's index of 0.7 , the optimal EJT policy provides $33 \%$ gain in efficiency as compared to the $\alpha$-fair policy for the case of $x_{\min }=0 \mathrm{~Kb} / \mathrm{s}$ and $21 \%$ gain in efficiency for the case of $x_{\min }=50 \mathrm{~Kb} / \mathrm{s}$.

In Figs. 5(a) and 5(b) we present the counterparts of Figs. 4(a) and 4(b), but for the case when the RBs are not time-shared by the users. In Fig. 5(a), $x_{\min }=0$, while in Fig. 5(b) $x_{\min }=50 \mathrm{~Kb} / \mathrm{s}$. Since the set $\mathcal{C}$ in this case is not convex and hence does not satisfy the sufficient conditions for possessing the monotonic tradeoff property, Procedure 1 was used to obtain the optimal EJT; cf. Section V-A. The nonconvex optimization problems underlying Procedure 1 and the $\alpha$-fair policy were solved using exhaustive search. Similar to the case of time-sharing considered in Figs. 4(a) and 4(b), it can be seen from Figs. 5(a) and 5(b) that, in this case too, the optimal EJT policy provides tradeoffs that are significantly better than those provided by the $\alpha$-fair policy when $x_{\min }=0$ and when $x_{\min }=50 \mathrm{~Kb} / \mathrm{s}$.

\section{B. Case 2: Ergodic Time-Varying Channel}

We now compare the performance of the $\alpha$-fair scheduler in (18) with the one proposed in (20). To do so, we consider a cellular network based on the IMT-advanced guidelines for the Urban Macro (UMa) scenario [23]. As per these guidelines, the number of hexagonal sectors is 57 , and these sectors are served by $19 \mathrm{BSs}$, each with a tri-sector antenna to serve a 3-sector cell-site. The users are uniformly dropped in the 57 sectors, and Monte Carlo simulations are carried over $10^{4}$ time slots and averaged over 10 independent drops. The users are assumed to be associated with the sector with the highest received average power. This power depends on large channel variations, which are mainly due to non-uniform antenna patterns (cf. [23, pp. 17-18]) and distance-dependent path-loss and correlated shadowing. To incorporate these variations in our simulations, we considered the line-of-sight (LOS) and non-line of-sight (NLOS) users as in [23, pp. 17-33]. For 


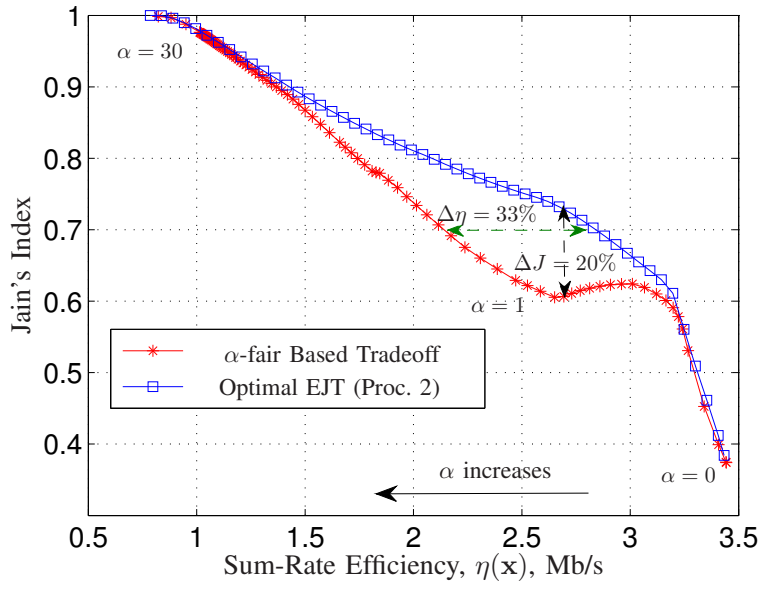

(a) $x_{\min }=0 \mathrm{~Kb} / \mathrm{s}$.

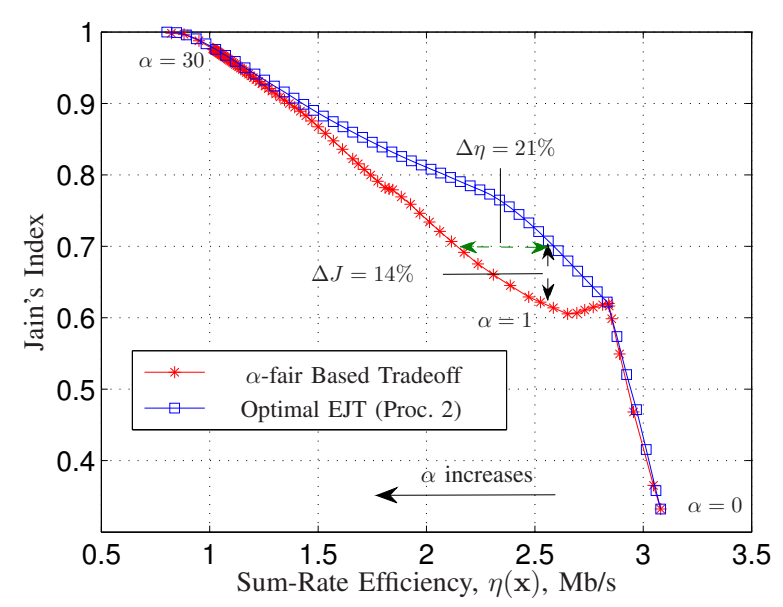

(b) $x_{\min }=50 \mathrm{~Kb} / \mathrm{s}$.

Fig. 4. A comparison between the EJTs achieved by the optimal and $\alpha$-fair policies, with time-sharing.

the LOS users, the path-loss exponent is set to be 2.2 and the shadowing is assumed log-normal with standard deviation of $4 \mathrm{~dB}$. For the NLOS users, the path-loss exponent is set to be 3.9 and the log-normal shadowing standard deviation is set to be $6 \mathrm{~dB}$. The system parameters are based on the LTE standard, whereby each RB is composed of a time slot of 7 OFDM symbols and 12 subcarriers [22]. The simulation parameters are given in Table I.

In addition to large variations, the received signal power is subject to small-scale variations due to time-varying multipath fading. Variations of the received signal power due to fading within each RB is negligible and hence, the channels can be assumed fixed over each RB. For other RBs the channels take on different values depending on the spectro-temporal correlation of the IMT-advanced model for the UMa scenario [24].

At time slot $t$, each user $m \in \mathcal{M}$ calculates its received signal-to-interference-plus-noise ratios (SINRs) on all the RBs, and subsequently determines $r_{m n}(t)$, the data rate that can be reliably communicated on every $\mathrm{RB} n \in \mathcal{N}$. The set of all the rates at time $t,\left\{r_{m n}(t)\right\}$, are available at the BS, which subsequently determines the appropriate scheduling variables,

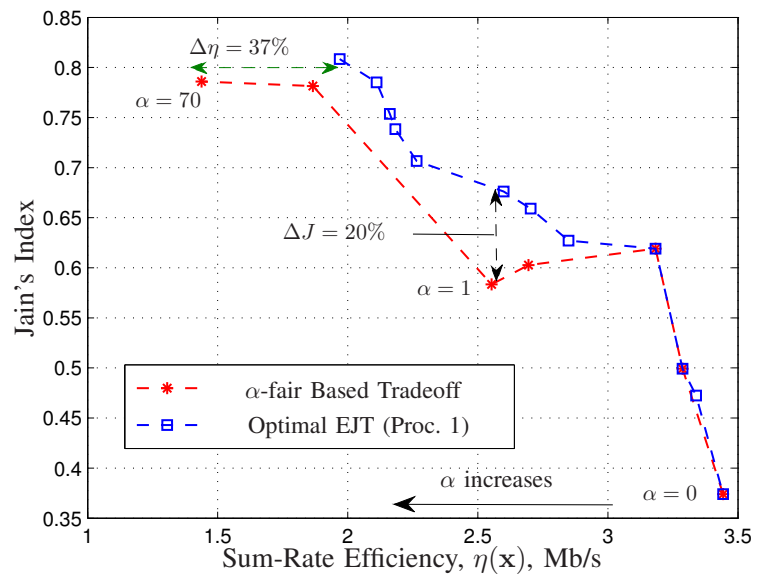

(a) $x_{\min }=0 \mathrm{~Kb} / \mathrm{s}$.

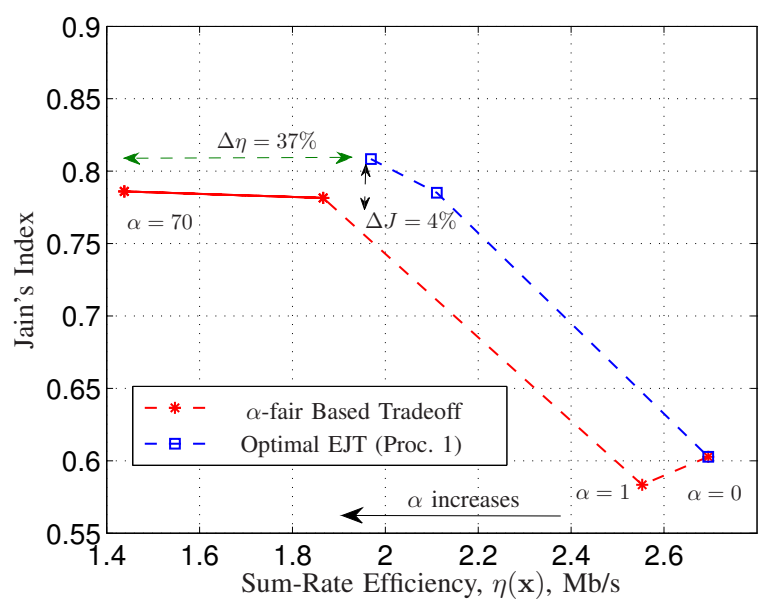

(b) $x_{\min }=50 \mathrm{~Kb} / \mathrm{s}$.

Fig. 5. A comparison between the EJTs achieved by the optimal and $\alpha$-fair policies, without time-sharing.

$\left\{\rho_{m n}(t)\right\}$, depending on previously scheduled rates and the adopted scheduling strategy.

1) EJT Comparison: Using the UMa model with the above parameter settings, in Fig. 6 we plot the EJT curve for both the $\alpha$-fair scheduler in (18) and the proposed scheduler in (20). From this figure it can be seen that the scheduler in (20) achieves better tradeoffs, especially in the high fairness region. For example, for a Jain's index of 0.94, the scheduler in (20) provides $33 \%$ gain in efficiency as compared to the $\alpha$-fair scheduler.

2) User Satisfaction Comparison: To illustrate the advantage of using Jain's index, we compare the number of satisfied users when the scheduling is performed based on the optimal EJT and $\alpha$-fair policies. In particular, we assume that a user is satisfied if its long-term average rate exceeds a certain threshold. To perform this comparison, for the $\alpha$-fair policy, the parameter $\alpha$ is chosen to maximize the percentage of users for which the rates obtained by the scheduler in (18) exceed a given threshold. Similarly, for the proposed optimal EJT policy, the parameter $\beta$ is chosen to maximize the percentage of users for which the rates obtained by the scheduler in (20) 
TABLE I

Simulation PARAMETERS BASED ON IMT-ADVANCED UMA SCENARIO.

\begin{tabular}{|c|c|}
\hline Parameter & Assumption or Value \\
\hline Number of sectors & $\overline{57}$ \\
\hline Number of users & 570 \\
\hline Inter-site distance & $500 \mathrm{~m}$ \\
\hline BS height & $25 \mathrm{~m}$ \\
\hline Min. distance $\mathrm{b} / \mathrm{w}$ user and $\mathrm{BS}$ & $25 \mathrm{~m}$ \\
\hline User speed & $30 \mathrm{~km} / \mathrm{h}$ \\
\hline Bandwidth (downlink) & $10 \mathrm{MHz}$ \\
\hline Sub-carrier spacing & $15 \mathrm{KHz}$ \\
\hline Number of RBs $(N)$ & 50 \\
\hline OFDM symbol duration & $66.67 \mu \mathrm{s}$ \\
\hline Number of sub-carriers per RB & 12 \\
\hline Number of OFDM symbols per RB & 7 \\
\hline Carrier Frequency $(\mathrm{GHz})$ & 2.0 \\
\hline Total BS transmit power & $46 \mathrm{dBm}$ \\
\hline Number of drops & 10 \\
\hline Number of time-slots per drop & 10000 \\
\hline Smoothing factor $(\mu)$ & 0.01 \\
\hline BS antenna gain (boresight) & $17 \mathrm{dBi}$ \\
\hline User antenna gain & $0 \mathrm{dBi}$ \\
\hline Feeder loss & $2 \mathrm{~dB}$ \\
\hline Channel estimation delay & 4 time slots \\
\hline SINR estimation margin & $6 \mathrm{~dB}$ \\
\hline Antenna tilt & $12^{\circ}[25$, p. 4$]$ \\
\hline Traffic model & Full buffer \\
\hline
\end{tabular}

exceed the same threshold. Unfortunately, obtaining closedform expressions for the optimal $\alpha$ and $\beta$ as functions of rate thresholds has proved intractable. As an alternative, for each threshold we perform an exhaustive search to find the optimal $\alpha$ and $\beta$ that maximize the number of satisfied users in each policy. In practice, the optimal $\alpha$ and $\beta$ can be determined through a pre-communication learning phase that enables the BSs to evaluate user satisfactions for tentative values of $\alpha$ and $\beta$.

The comparison between the percentage of satisfied users at given rate thresholds is plotted in Fig. 7. As expected, the number of satisfied users is monotonically decreasing with the threshold for both schedulers. However, the scheduler based on the optimal EJT is consistently better than the one based on the $\alpha$-fair policy as it enables significantly more users to be satisfied. For instance, for the considered scenario when the threshold is set to be $1 \mathrm{Mb} / \mathrm{s}$, the scheduler based on the optimal EJT satisfies $10 \%$ more users than those satisfied by the scheduler based on the $\alpha$-fair policy. Hence, from a service provider's perspective, using the scheduler based on the optimal EJT yields valuable increase in the ability of the system to satisfy users with long-term average rate requirements.

\section{CONCLUSIONS}

In this paper, we considered multiuser resource allocations that achieve the optimal tradeoff between efficiency and fairness from the Jain's index perspective. We showed that, in general, the commonly-used $\alpha$-fair policy does not yield the optimal EJTs except for the two-user case. To achieve the optimal EJTs in the general case, we developed two procedures. In the first procedure, the set of feasible allocations is arbitrary, but finding the allocations that achieve the optimal

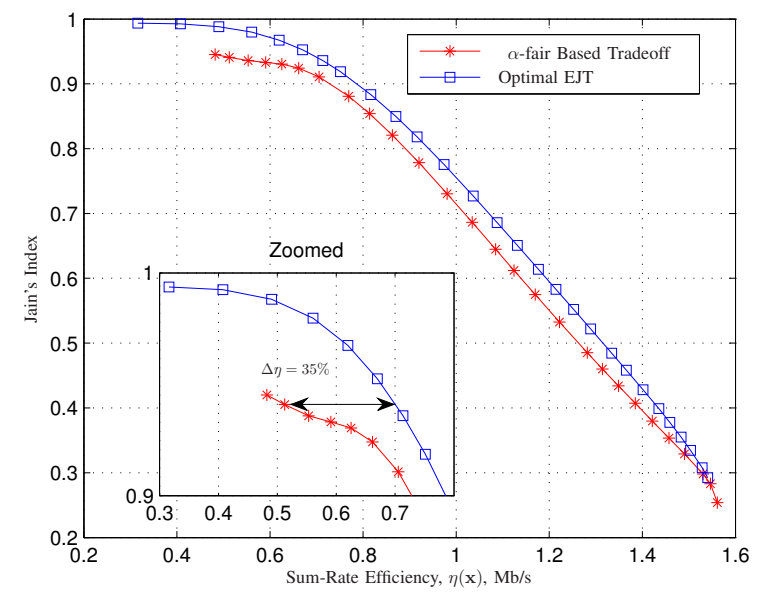

Fig. 6. A comparison between the EJTs achieved by the optimal and $\alpha$-fair policies in the long-term average rates.

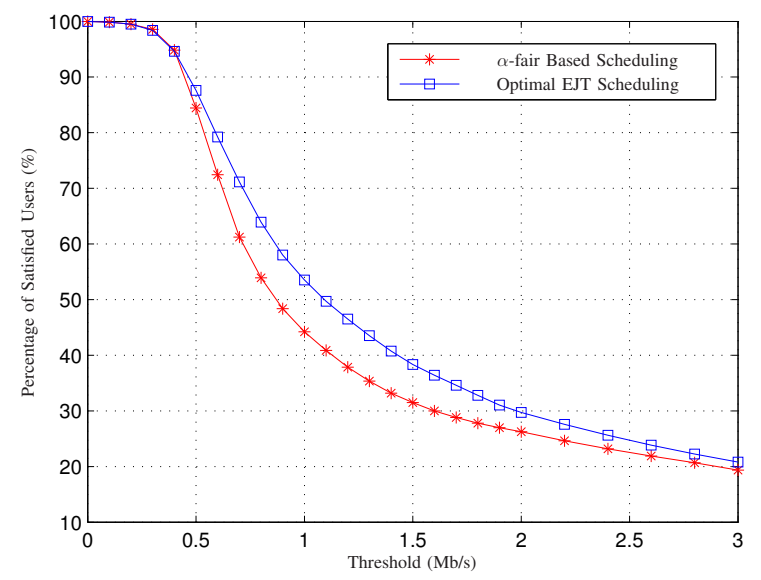

Fig. 7. Percentage of satisfied users for different thresholds.

EJTs involves solving potentially difficult optimization problems. In contrast, in the second procedure, the set of feasible allocations is assumed to have a monotonic property that arises in many practical scenarios. This property is exploited to facilitate the search for allocations that achieve the optimal EJTs. This property is shown to arise naturally in the problem of scheduling radio resources in quasi-static and ergodic timevarying communication scenarios, and it enables us to devise computationally-efficient schedulers that achieve the optimal EJT. Our analysis is supported by illustrations, geometric interpretations and numerical examples.

\section{ACKNOWLEDGMENT}

The authors would like to express their gratitude to Dr. Gamini Senarath, Dr. Ho Ting Cheng, and Dr. Petar Djukic of Huawei Technologies Canada Co., Ltd.

\section{APPENDIX A}

\section{Proof of PROPOSITION 1}

We will proceed by contradiction. Let $\alpha \in(0, \infty)$ be given and suppose that $\mathbf{x}_{\alpha}^{\star}$ does not achieve the optimal EJT, that is, 
there exists a non $\alpha$-fair optimal vector $\mathrm{x}$ such that either 1) $\eta(\mathbf{x})>\eta\left(\mathbf{x}_{\alpha}^{\star}\right)$ and $J(\mathbf{x}) \geq J\left(\mathbf{x}_{\alpha}^{\star}\right)$; or 2) $\eta(\mathbf{x}) \geq \eta\left(\mathbf{x}_{\alpha}^{\star}\right)$ and $J(\mathbf{x})>J\left(\mathbf{x}_{\alpha}^{\star}\right)$. We will show that such a vector $\mathbf{x}$ results in $U_{\alpha}(\mathbf{x})>U_{\alpha}\left(\mathbf{x}_{\alpha}^{\star}\right)$, which contradicts the definition of $\alpha$-fair benefit vectors; cf. (2). We will focus on the first case. The proof for the second case follows similar lines and is omitted for brevity.

Since $M=2$, we can define a parameter $\omega=\frac{\max \mathbf{x}}{\min \mathbf{x}}$. Using this $\omega$, we have $J(\mathbf{x})=\frac{(1+\omega)^{2}}{2\left(1+\omega^{2}\right)}$. Now, $\frac{d J}{d \omega}=-\frac{\omega^{2}-1}{\left(\omega^{2}+1\right)^{2}}$. Since, by definition, $\omega \geq 1$, it can be seen that $J$ is monotonically decreasing in $\omega$. This with the fact that, in the considered case, $J(\mathbf{x}) \geq J\left(\mathbf{x}_{\alpha}^{\star}\right)$ implies that

$$
\frac{\max \mathbf{x}}{\min \mathbf{x}} \leq \frac{\max \mathbf{x}_{\alpha}^{\star}}{\min \mathbf{x}_{\alpha}^{\star}}
$$

Since in this case we also have $\eta(\mathbf{x})>\eta\left(\mathbf{x}_{\alpha}^{\star}\right)$, it follows that $\min \mathbf{x}+\max \mathbf{x}>\min \mathbf{x}_{\alpha}^{\star}+\max x_{\alpha}^{\star}$, which is equivalent to $\left(1+\frac{\max \mathbf{x}}{\min \mathbf{x}}\right) \min \mathbf{x}>\left(1+\frac{\max \mathbf{x}_{\alpha}^{\star}}{\min \mathbf{x}_{\alpha}^{\star}}\right) \min \mathbf{x}_{\alpha}^{\star}$. This inequality implies that

$$
\min \mathbf{x}>\frac{\left(1+\frac{\max \mathbf{x}_{\alpha}^{\star}}{\min \mathbf{x}_{\alpha}^{\star}}\right)}{\left(1+\frac{\max \mathbf{x}}{\min \mathbf{x}}\right)} \min \mathbf{x}_{\alpha}^{\star} .
$$

Invoking (22) implies that the fraction on the right hand side is greater than 1 , which further implies that we can write $\min \mathbf{x}=\min \mathbf{x}_{\alpha}^{\star}+\epsilon_{1}$, with $\epsilon_{1}>0$. Since $\mathbf{x}$ is not $\alpha$-fair, we must have

$$
U_{\alpha}(\mathbf{x})<U_{\alpha}\left(\mathbf{x}_{\alpha}^{\star}\right)
$$

We now observe that $U_{\alpha}(\mathbf{x})$ is strictly increasing in each $x_{m}$, $m=1,2$. This observation and (24) imply that $\max \mathbf{x}=$ $\max \mathbf{x}_{\alpha}^{\star}-\epsilon_{2}$, with $\epsilon_{2}>0$. Combining this with the fact that $\min \mathbf{x}=\min \mathbf{x}_{\alpha}^{\star}+\epsilon_{1}$ and the fact that in the current case $\eta(\mathbf{x})>\eta\left(\mathbf{x}_{\alpha}^{\star}\right)$ yields $\epsilon_{1}>\epsilon_{2}$. Using this notation, it can be readily verified that, because $\epsilon_{1}>\epsilon_{2}$,

$$
\begin{aligned}
\nabla U_{\alpha}(\mathbf{x})^{T}\left(\mathbf{x}_{\alpha}^{\star}-\mathbf{x}\right) & =-\epsilon_{1}(\min \mathbf{x})^{-\alpha}\left(1-\frac{\epsilon_{2}}{\epsilon_{1}}\left(\frac{\max \mathbf{x}}{\min \mathbf{x}}\right)^{-\alpha}\right) \\
& <0
\end{aligned}
$$

Now, direct computation of the Hessian of $U_{\alpha}(\mathbf{x})$ shows that $U_{\alpha}$ is concave for any $\alpha \in(0, \infty)$. Thus [14, p. 69], $U_{\alpha}\left(\mathbf{x}_{\alpha}^{\star}\right) \leq U_{\alpha}(\mathbf{x})+\nabla U_{\alpha}(\mathbf{x})^{T}\left(\mathbf{x}_{\alpha}^{\star}-\mathbf{x}\right)$, which yields

$$
U_{\alpha}\left(\mathbf{x}_{\alpha}^{\star}\right)<U_{\alpha}(\mathbf{x}) .
$$

This with (24) establish the desired contradiction.

\section{APPENDIX B \\ PROOF OF THEOREM 1}

Let $\mathrm{x}_{\sigma_{1}}^{\star}$ and $\mathrm{x}_{\sigma_{2}}^{\star}$ be the benefit vectors obtained using (10) with $\sigma_{1}$ and $\sigma_{2}$, respectively, where $\sigma_{\min } \leq \sigma_{1}<\sigma_{2} \leq \sigma_{\max }$. To prove Theorem 1, it suffices to show that if the conditions of the theorem are satisfied, then $J_{\sigma_{1}}^{\star} \geq J_{\sigma_{2}}^{\star}$ with equality if and only if $J_{\sigma_{2}}^{\star}=J_{\sigma^{\star}}^{\star}$, where $J_{\sigma}^{\star}$ and $J_{\sigma^{\star}}^{\star}$ are given by (8) and (9), respectively. We consider two distinct cases: $x_{\min }>0$ and $x_{\min }=0$.
Case $1\left(x_{\min }>0\right)$ : First we note that, because $x_{\min } \mathbf{1} \in$ $\mathcal{C}, J_{\sigma^{\star}}^{\star}=1$. Now, the convexity of $\mathcal{C}$ implies that, for any $\theta \in(0,1)$,

$$
\mathbf{y}_{\theta}=\theta x_{\min } \mathbf{1}+(1-\theta) \mathbf{x}_{\sigma_{2}}^{\star} \in \mathcal{C} .
$$

Substituting for $\mathbf{y}_{\theta}$ from (26) in (1), it can be verified that

$$
J\left(\mathbf{y}_{\theta}\right)-J_{\sigma_{2}}^{\star}=a\left(1-J\left(\mathbf{y}_{\theta}\right)\right),
$$

where $a=\frac{\theta^{2} M^{2} x_{\min }^{2}+2 \theta(1-\theta) M x_{\min } \mathbf{1}^{T} \mathbf{x}_{\sigma}^{\star}}{M(1-\theta)^{2} \mathbf{x}_{\sigma}^{\star T} \mathbf{x}_{\sigma}^{\star}} \geq 0$.

To prove the theorem, we will show that $J\left(\mathbf{y}_{\theta}\right)-J_{\sigma_{2}}^{\star} \geq 0$, with equality if and only if $J_{\sigma_{2}}^{\star}=1$. To do so, we note that, by the properties of Jain's index, if $J_{\sigma_{2}}^{\star}<1$, then $\mathbf{x}_{\sigma_{2}}^{\star} \neq \gamma_{1} \mathbf{1}$, and subsequently from (26) $\mathbf{y}_{\theta} \neq \gamma_{2} \mathbf{1}$ for any $\gamma_{1}, \gamma_{2}>0$. This implies that $J\left(\mathbf{y}_{\theta}\right)<1$, which further implies from (27) and the fact that $a>0$ for any $\theta \in(0,1)$ that $J\left(\mathbf{y}_{\theta}\right)>J_{\sigma_{2}}^{\star}$. We next show that there exists a $\theta_{0} \in(0,1)$ such that $\eta\left(\mathbf{y}_{\theta_{0}}\right)=\sigma_{1}$. In particular, setting $\theta_{0}=\frac{\sigma_{2}-\sigma_{1}}{\sigma_{2}-M x_{\min }}$ yields this efficiency. Since $J_{\sigma_{1}}^{\star}$ is the maximum Jain's index corresponding to $\eta(\mathbf{x})=\sigma_{1}$, we must have $J_{\sigma_{1}}^{\star} \geq J\left(\mathbf{y}_{\theta_{0}}\right)$, which implies that $J_{\sigma_{1}}^{\star}>J_{\sigma_{2}}^{\star}$.

For the case of $J_{\sigma_{2}}^{\star}=1$, we note that $\mathbf{y}_{\theta}=\gamma_{2} \mathbf{1}$, for some $\gamma_{2}$ and therefore, by the homogeneity of Jain's index, $J\left(\mathbf{y}_{\theta}\right)=1$ for any $\theta$. The statement of the theorem follows by using an argument analogous to the above and noting that $J_{\sigma}^{\star} \leq 1$, for any $\sigma$ including $\sigma_{1}$.

Case $2\left(x_{\min }=0\right)$ : The proof for the case of $x_{\min }>0$ does not hold when $x_{\min }=0$. Furthermore, it is easy to find sets $\mathcal{C}$ for which $x_{\text {min }}=0$, but vectors of the form $\gamma_{1} \mathbf{1} \notin \mathcal{C}$ even for infinitesimal values of $\gamma_{1}>0$, e.g., $\mathcal{C}=\left\{x_{1}, x_{2}\right.$ : $\left.0 \leq x_{2} \leq c x_{1}\right\}$, where $c \in(0,1)$.

To prove the theorem for this case, we provide the following lemma:

Lemma 1. Let $\mathcal{C}$ be a set satisfying the conditions of Theorem 1 with $x_{\min }=0$ and let $\sigma_{\min } \leq \sigma_{1} \leq \sigma_{2} \leq \sigma_{\max }$, then $J_{\sigma_{1}}^{\star} \geq J_{\sigma_{2}}^{\star}$.

Before providing the proof, we note that unlike the claim of Theorem 1, this lemma does not tell whether a strict decrease in $\sigma$ will yield a strict increase in Jain's index.

Proof: Let $\mathrm{x}_{\sigma_{2}}^{\star}$ be the optimal benefit vector at efficiency $\sigma_{2}$; that is, $J\left(\mathbf{x}_{\sigma_{2}}^{\star}\right)=J_{\sigma_{2}}^{\star}$. Since $\mathcal{C}$ is convex and $\mathbf{0}_{M} \in \mathcal{C}$, it follows that, for any $\theta \in[0,1], \hat{\mathbf{x}}=\theta \mathbf{x}_{\sigma_{2}}^{\star} \in \mathcal{C}$. Noting that $\sigma_{1} \leq \sigma_{2}$ and setting $\theta=\frac{\sigma_{1}}{\sigma_{2}}$, it can be seen that $\eta(\hat{\mathbf{x}})=\sigma_{1}$. Now, the homogeneity of Jain's index implies that $J(\hat{\mathbf{x}})=$ $J_{\sigma_{2}}^{\star}$. However, $J_{\sigma_{1}}^{\star}$ is the optimal Jain's index at efficiency $\sigma_{1}$ and hence $J_{\sigma_{1}}^{\star} \geq J(\hat{\mathbf{x}})$, which completes the proof of the lemma.

We now proceed to complete the proof of Theorem 1. Let $\sigma^{\star}$ be the highest efficiency corresponding to the maximum achievable Jain's index, i.e., $J_{\sigma^{\star}}^{\star}$. We will consider two cases: $\sigma_{2} \leq \sigma^{\star}$ and $\sigma_{2}>\sigma^{\star}$.

When $\sigma_{2} \leq \sigma^{\star}$, we have by assumption that $\sigma_{1}<\sigma^{\star}$, which, by Lemma 1 , implies that $J_{\sigma_{2}}^{\star}=J_{\sigma_{1}}^{\star}=J_{\sigma_{2}}^{\star}$, and the theorem is proved in this case.

We now consider the case of $\sigma_{2}>\sigma^{\star}$. We again consider two cases: $\sigma_{1} \leq \sigma^{\star}$ and $\sigma_{1}>\sigma^{\star}$. 
Since $\sigma_{2}>\sigma^{\star}$, it follows by the definition of $\sigma^{\star}$ that $J_{\sigma_{2}}^{\star}<$ $J_{\sigma^{\star}}^{\star}$. Now, if $\sigma_{1} \leq \sigma^{\star}$, invoking Lemma 1 yields $J_{\sigma_{1}}^{\star}=J_{\sigma^{\star}}^{\star}$ which implies that $J_{\sigma_{2}}^{\star}<J_{\sigma_{1}}^{\star}$.

We next prove the theorem for the remaining case of $\sigma_{2}>$ $\sigma_{1}>\sigma^{\star}$.

For this case, we will consider the line segment, $\mathbf{y}_{\theta}$, connecting $\mathbf{x}_{\sigma_{2}}^{\star}$ with $\mathbf{x}_{\sigma_{\star}}^{\star}$, i.e., $\mathbf{y}_{\theta}=\theta \mathbf{x}_{\sigma_{\star}}^{\star}+(1-\theta) \mathbf{x}_{\sigma_{2}}^{\star}$, $\theta \in[0,1]$. The convexity assumption implies that $\mathbf{y}_{\theta} \in \mathcal{C}$. Substituting for $\mathbf{y}_{\theta}$ in (1), it can be verified that, for any $\mathbf{x}_{\sigma_{2}}^{\star}, \mathbf{y}_{\theta}$ such that $\left\|\mathbf{x}_{\sigma_{2}}^{\star}\right\|\left\|\mathbf{y}_{\theta}\right\|>0$,

$$
\begin{aligned}
& J\left(\mathbf{y}_{\theta}\right)-J\left(\mathbf{x}_{\sigma_{2}}^{\star}\right)= \\
& \frac{\theta^{2}\left(\sigma^{\star 2}\left\|\mathbf{x}_{\sigma_{2}}^{\star}\right\|^{2}-\sigma_{2}^{2}\left\|\mathbf{x}_{\sigma_{\star}}^{\star}\right\|^{2}\right)+2 \theta(1-\theta) \sigma_{2}\left(\sigma^{\star}\left\|\mathbf{x}_{\sigma_{2}}^{\star}\right\|^{2}-\sigma_{2} \mathbf{x}_{\sigma^{\star}}^{\star} \mathbf{x}_{\sigma_{2}}^{\star}\right)}{M\left\|\mathbf{x}_{\sigma_{2}}^{\star}\right\|^{2}\left\|\mathbf{y}_{\theta}\right\|^{2}} .
\end{aligned}
$$

We will now use this equality to show that $J\left(\mathbf{y}_{\theta}\right)>J\left(\mathbf{x}_{\sigma_{2}}^{\star}\right)$. For the first term in the numerator, we note that, since by assumption $\sigma_{2}>\sigma^{\star}$, we have $J_{\sigma_{2}}^{\star}<J_{\sigma^{\star}}^{\star}$, which implies that $\sigma^{\star 2}\left\|\mathbf{x}_{\sigma_{2}}^{\star}\right\|^{2}-\sigma_{2}^{2}\left\|\mathbf{x}_{\sigma_{\star}}^{\star}\right\|^{2}>0$. For the second term, we note that, by the Cauchy-Schwartz inequality,

$$
\sigma^{\star}\left\|\mathbf{x}_{\sigma_{2}}^{\star}\right\|^{2}-\sigma_{2} \mathbf{x}_{\sigma^{\star}}^{\star}{ }^{T} \mathbf{x}_{\sigma_{2}}^{\star} \geq\left\|\mathbf{x}_{\sigma_{2}}^{\star}\right\|\left(\sigma^{\star}\left\|\mathbf{x}_{\sigma_{2}}^{\star}\right\|-\sigma_{2}\left\|\mathbf{x}_{\sigma^{\star}}^{\star}\right\|\right)>0 .
$$

The strict positivity follows from the fact that $J_{\sigma_{2}}^{\star}<J_{\sigma^{\star}}^{\star}$. Hence, we have shown that $J\left(\mathbf{y}_{\theta}\right)>J\left(\mathbf{x}_{\sigma_{2}}^{\star}\right)$ for any $\theta \in$ $(0,1)$.

We next show that there exists a $\theta_{0} \in(0,1)$ such that $\eta\left(\mathbf{y}_{\theta_{0}}\right)=\sigma_{1}$. In particular, setting $\theta_{0}=\frac{\sigma_{2}-\sigma_{1}}{\sigma_{2}-\sigma^{\star}}$ yields this efficiency, where $\theta_{0} \in(0,1)$ by the assumption that $\sigma_{1}>\sigma^{\star}$. Since $J_{\sigma_{1}}^{\star}$ is the maximum Jain's index corresponding to $\eta(\mathbf{x})=\sigma_{1}$, we must have $J_{\sigma_{1}}^{\star} \geq J\left(\mathbf{y}_{\theta_{0}}\right)$, which implies that $J_{\sigma_{1}}^{\star}>J_{\sigma_{2}}^{\star}$ and completes the proof of the theorem.

\section{REFERENCES}

[1] J. Mo and J. Walrand, "Fair end-to-end window-based congestion control," IEEE/ACM Trans. Netw., vol. 8, no. 5, pp. 556-567, Oct. 2000.

[2] T. Lan, D. Kao, M. Chiang, and A. Sabharwal, "An axiomatic theory of fairness in network resource allocation," in Proc. IEEE Int. Conf. Comp. Commun. (INFOCOM), San Diego, California, Mar. 2010.

[3] H. T. Cheng and W. Zhuang, "An optimization framework for balancing throughput and fairness in wireless networks with QoS support," IEEE Trans. Wireless Commun., vol. 7, no. 2, pp. 584-593, Feb. 2008.

[4] R. Schoenen, A. Bin Sediq, H. Yanikomeroglu, G. Senarath, and Z. Chao, "Fairness analysis in cellular networks using stochastic Petri nets," in Proc. IEEE Int. Symp. on Pers., Indoor and Mobile Radio Commun. (PIMRC), Toronto, Canada, Sep. 2011.

[5] R. Schoenen, A. Bin Sediq, H. Yanikomeroglu, G. Senarath, Z. Chao, and H. T. Cheng, "Spectral efficiency and fairness tradeoffs in cellular networks with realtime+nonrealtime traffic mix using stochastic Petri nets," in Proc. IEEE Veh. Tech. Conf. (VTC-Fall), Quebec City, Canada, Sep. 2012.

[6] D. Bertsekas and R. Gallager, Data Networks, 2nd ed. Prentice Hall, 1992.

[7] A. Odlyzko, "Network neutrality, search neutrality, and the neverending conflict between efficiency and fairness in markets," Rev. Ntwk. Economics, vol. 8, no. 1, pp. 40-60, Mar. 2009.

[8] M. Zukerman, L. Tan, H. Wang, and I. Ouveysi, "Efficiency-fairness tradeoff in telecommunications networks," IEEE Commun. Lett., vol. 9, no. 7, pp. 643-645, Jul. 2005.

[9] R. Jain, D. Chiu, and W. Hawe, "A quantitative measure of fairness and discrimination for resource allocation in shared systems," Digital Equipment Corporation, DEC-TR-301, Tech. Rep., 1984. [Online]. Available: http://www1.cse.wustl.edu/ jain/papers/ftp/fairness.pdf.

[10] F. P. Kelly, A. K. Maulloo, and D. K. H. Tan, "Rate control for communication networks: Shadow prices, proportional fairness and stability," J. Oper. Research Scty., vol. 49, no. 3, pp. 237-252, 1998.
[11] C. Wengerter, J. Ohlhorst, and A. von Elbwart, "Fairness and throughput analysis for generalized proportional fair frequency scheduling in OFDMA," in Proc. IEEE Veh. Technol. Conf., vol. 3, May 2005, pp. 1903-1907.

[12] C. Joe-Wong, S. Sen, T. Lan, and M. Chiang, "Multi-resource allocation: Fairness-efficiency tradeoffs in a unifying framework," in Proc. IEEE Int. Conf. Comp. Commun. (INFOCOM), Orlando, Florida, Mar. 2012.

[13] A. Ibing and H. Boche, "Fairness vs. efficiency: Comparison of game theoretic criteria for OFDMA scheduling." in Proc. Asilomar Conf. Signals, Systems \& Computers, Nov. 2007, pp. 275-279.

[14] S. Boyd and L. Vandenberghe, Convex Optimization. Cambridge University Press, 2004.

[15] A. Bin Sediq, R. H. Gohary, and H. Yanikomeroglu, "Optimal tradeoff between efficiency and Jain's fairness index in resource allocation," in Proc. IEEE Int. Symp. on Pers., Indoor and Mobile Radio Commun. (PIMRC), Sydney, Australia, Sep. 2012.

[16] B. Radunovic and J.-Y. L. Boudec, "Rate performance objectives of multihop wireless networks," IEEE Trans. Mobile Comput., vol. 3, no. 4 pp. 334-349, Oct. 2004.

[17] G. Song and Y. Li, "Cross-layer optimization for OFDM wireless networks-part II: Algorithm development," IEEE Trans. Wireless Commun., vol. 4, no. 2, pp. 625-634, Mar. 2005.

[18] E. Biglieri, J. Proakis, and S. Shamai (Shitz), "Fading channels: Information-theoretic and communication aspects," IEEE Trans. Inf. Theory, vol. 44, no. 6, pp. 2619-2692, Oct. 1998.

[19] C. Y. Wong, R. S. Cheng, K. Ben Letaief, and R. D. Murch, "Multiuser OFDM with adaptive subcarrier, bit, and power allocation," IEEE $J$. Select. Areas Commun., vol. 17, no. 10, pp. 1747-1758, Oct. 1999.

[20] A. L. Stolyar, "On the asymptotic optimality of the gradient scheduling algorithm for multiuser throughput allocation," Oper. Research, vol. 53, no. 1, pp. 12-25, Jan. 2005.

[21] H. Kushner and P. Whiting, "Convergence of proportional-fair sharing algorithms under general conditions," IEEE Trans. Wireless Commun., vol. 3, no. 4, pp. 1250-1259, Jul. 2004.

[22] E. Dahlman, S. Parkvall, J. Skold, and P. Beming, $3 G$ Evolution: HSPA and LTE for Mobile Broadband, 2nd ed. Academic Press, 2008.

[23] ITU, Report ITU-R M.2135-1, "Guidelines for evaluation of radio interface technologies for IMT-Advanced," ITU, Tech. Rep., Dec. 2009. [Online]. Available: http://www.itu.int/pub/R-REP-M.2135-1-2009.

[24] J. Nystrom, "Software implementation of IMT.Eval channel model," CELTIC/CP5-026 Project WINNER+ Doc. 5D/478-E, Tech. Rep., Jul. 2009. [Online]. Available: http://www.itu.int/oth/R0A06000022/en.

[25] "Calibration for IMT-Advanced evaluations," CELTIC/CP5-026 Project WINNER+, Tech. Rep., May 2010. [Online]. Available: http://projects. celtic-initiative.org/winner+/WINNER+EvaluationGroup.html. 